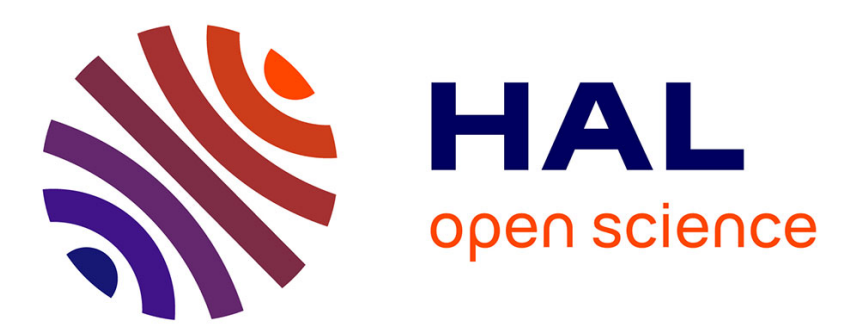

\title{
Genetic, Biochemical, and Structural Characterization of CMY-136 Beta-Lactamase, a Peculiar CMY-2 Variant
}

Agustin Zavala, Pascal Retailleau, Eddy Elisée, Bogdan Iorga, Thierry Naas

\section{To cite this version:}

Agustin Zavala, Pascal Retailleau, Eddy Elisée, Bogdan Iorga, Thierry Naas. Genetic, Biochemical, and Structural Characterization of CMY-136 Beta-Lactamase, a Peculiar CMY-2 Variant. ACS Infectious Diseases, 2019, 5 (4), pp.528-538. 10.1021/acsinfecdis.8b00240 . hal-02119851

\section{HAL Id: hal-02119851 \\ https://hal.science/hal-02119851}

Submitted on 10 Mar 2021

HAL is a multi-disciplinary open access archive for the deposit and dissemination of scientific research documents, whether they are published or not. The documents may come from teaching and research institutions in France or abroad, or from public or private research centers.
L'archive ouverte pluridisciplinaire HAL, est destinée au dépôt et à la diffusion de documents scientifiques de niveau recherche, publiés ou non, émanant des établissements d'enseignement et de recherche français ou étrangers, des laboratoires publics ou privés. 


\section{Genetic, biochemical and structural characterization of CMY-136 $\beta$-lactamase, a peculiar CMY-2 variant}

Agustin Zavala ${ }^{1,2}$, Pascal Retailleau ${ }^{1}$, Eddy Elisée ${ }^{1}$, Bogdan I. Iorga $^{1 *}$, Thierry Naas ${ }^{2,3,4,5^{*}}$

${ }^{1}$ Institut de Chimie des Substances Naturelles, CNRS UPR 2301, Université Paris-Saclay, LabEx

LERMIT, 1 avenue de la Terrasse, Bât. 27, 91198 Gif-sur-Yvette, France

${ }^{2}$ EA7361 "Structure, dynamic, function and expression of broad spectrum $\beta$-lactamases", Université Paris Sud, Université Paris Saclay, LabEx LERMIT, Faculty of Medicine, 78 rue du Général Leclerc, 94275 Le Kremlin-Bicêtre, France

${ }^{3}$ Bacteriology-Hygiene unit, Assistance Publique/Hôpitaux de Paris, Bicêtre Hospital, 78 rue du Général Leclerc, 94275 Le Kremlin-Bicêtre, France

${ }^{4}$ Associated French National Reference Center for Antibiotic Resistance: Carbapenemaseproducing Enterobacteriaceae, 78 rue du Général Leclerc, 94275 Le Kremlin-Bicêtre, France

${ }^{5}$ Evolution and Ecology of Resistance to Antibiotics Unit, Institut Pasteur - APHP -Université Paris Sud, 25-28 Rue du Dr Roux, 75015 Paris, France

*To whom correspondence should be addressed:

Bogdan I. Iorga: Institut de Chimie des Substances Naturelles, CNRS UPR 2301, 91198 Gif-surYvette, France. E-mail: bogdan.iorga@cnrs.fr; Tel. +33 1698230 94; Fax. +33 169077247

Thierry Naas : Service de Bactériologie-Hygiène, Hôpital de Bicêtre, 78 rue du Général Leclerc, 94275 Le Kremlin-Bicêtre, France. E-mail: thierry.naas@aphp.fr; Tel: +33 1452120 19; Fax: +33 145216340

Keywords: beta-lactamase, crystal structure, docking, molecular dynamics, antibiotic resistance, CMY-2, cephalosporinase, ESAC, CAZ, TIC, $\Omega$-loop 
With the widespread use and abuse of antibiotics for the past decades, antimicrobial resistance poses a serious threat to public health nowadays. $\beta$-Lactams are the most used antibiotics, and $\beta$ lactamases the most widespread resistance mechanism. Class $\mathrm{C} \beta$-lactamases, also known as cephalosporinases, usually do not hydrolyse the latest and most potent $\beta$-lactams, expanded spectrum cephalosporins and carbapenems. However, the recent emergence of extended-spectrum AmpC cephalosporinases, their resistance to inhibition by classic $\beta$-lactamase inhibitors, and the fact that they can contribute to carbapenem resistance when paired with impermeability mechanisms, means that these enzymes may still prove worrisome in the future. Here we report and characterize the CMY-136 $\beta$-lactamase, a Y221H point mutant derivative of CMY-2. CMY-136 confers an increased level of resistance to ticarcillin, cefuroxime, cefotaxime and ceftolozane/tazobactam. It is also capable of hydrolysing ticarcillin and cloxacillin, which act as inhibitors of CMY-2. X-ray crystallography and modelling experiments suggest that the hydrolytic profile alterations seem to be the result of an increased flexibility and altered conformation of the $\Omega$-loop, caused by the Y221H mutation. 
CMY-2-like enzymes are plasmidic class C $\beta$-lactamases that originated from the chromosomallyencoded AmpC of Citrobacter freundii ${ }^{1}$. They can be found in humans, feedstock animals, and pets $^{2}$. CMY-2 is the most widespread plasmid-mediated AmpC (pAmpC) $\beta$-lactamase, which can be

illustrated by the CMY-2-like enzymes reported to date, which amount to over $140^{3}$. They are also known as cephamycinases, because of their potential to hydrolyse these substrates, but they are also active on penicillins and first generation cephalosporins, and fairly resistant to inhibition by classical $\beta$-lactamase inhibitors (clavulanic acid, tazobactam and sulbactam) ${ }^{1}$. They are less active against third or fourth generation cephalosporins, and almost completely inactive against carbapenems. Lately however, several class C enzymes with extended-spectrum activity, termed extended-spectrum AmpC cephalosporinases (ESACs) ${ }^{4}$, have been reported, among them members of CMY-2-like family. Many of these enzymes show increased activity against third generation cephalosporins such as ceftazidime or cefotaxime, and even cefepime, a fourth-generation cephalosporin. When paired with impermeability mechanisms, CMY-2 may even mediate carbapenem resistance 5 . These enzymes are of clinical importance, and their study may prove important not only in understanding how their hydrolysis profile might evolve, but also to gain insights into their structure-function relationship, which in turn may aid in the development of new inhibitors, not only for class $\mathrm{C}$ but also for other classes of $\beta$-lactamases. We report here a novel CMY-2-like $\beta$-lactamase, CMY-136, we characterize it both biochemically and structurally, and provide the possible structural basis behind its hydrolysis profile differences when compared to CMY-2.

\section{RESULTS}

\section{Isolation of bla $a_{\mathrm{CMY}-136}$ gene}

E. coli EC13 clinical isolate, identified from a urinary tract infection, displayed an unusual cephalosporinase phenotype ${ }^{6}$. This isolate was resistant to ticarcillin and expanded spectrum cephalosporins, showed reduced susceptibility to cefoxitin, and showed increased susceptibility to 
inhibition by clavulanic acid. Performing susceptibility testing on cloxacillin containing plates restored susceptibility to ticarcillin and expanded spectrum cephalosporins, suggesting a class $\mathrm{C}$ enzyme. PCR was positive for CMY-2, and sequencing revealed a novel CMY-2-like variant, which was assigned the name CMY-136, differing by a single amino acid substitution, Y221H, in a highly conserved position in class C $\beta$-lactamases ${ }^{3}$. The sequence alignment between CMY-2 and CMY136 is shown in Fig. S1.

\section{Phenotypical characterization of CMY-136}

The antimicrobial susceptibility profile conferred by CMY-136 was compared to that of CMY-2 by transforming E. coli TOP10 cells with a pTOPO plasmid harbouring either blaCMY-2 or blaCMY136 genes and performing disk diffusion susceptibility tests and MICs assays. CMY-2 and CMY136 show similar resistance profiles, they both confer resistance to penicillins and cephalosporins, and show susceptibility to cefepime and carbapenems (see Fig. S2 for the chemical structures of these $\beta$-lactams). Based on the disk diffusion test, when compared to CMY-2, CMY-136 confers increased resistance to ticarcillin and cefotaxime, low level resistance to cefoxitin, and shows increased susceptibility to inhibition by clavulanic acid. The MICs show that when compared to CMY-2, CMY-136 confers to E. coli decreased resistance to piperacillin and cefoxitin, and an increased level of resistance to ticarcillin, cefuroxime and cefotaxime (Table 1). According to the breakpoints of EUCAST, E. coli TOP10 expressing CMY-136 is also resistant to ceftolozane/tazobactam, whereas under the same conditions CMY-2 confers no resistance to it.

Table 1 MICs for E. coli TOP10 pTOPO-CMY-2 and E. coli TOP10 pTOPO-CMY-136.

\begin{tabular}{|c|c|c|c|c|c|c|}
\hline \multirow{2}{*}{$\beta$-lactam } & \multicolumn{3}{|c|}{ E. coli TOP10 pTOPO-CMY-2 } & \multicolumn{3}{|c|}{ E. coli TOP10 pTOPO-CMY-136 } \\
\hline & $\mathrm{MIC}(\mathrm{g} / \mathrm{L})$ & CLSI & EUCAST & $\mathrm{MIC}(\mathrm{g} / \mathrm{L})$ & CLSI & EUCAST \\
\hline Amoxicillin & $>256$ & $-*$ & $\mathrm{R}$ & $>256$ & $-*$ & $\mathrm{R}$ \\
\hline Amoxicillin + CLA $^{a}$ & 32 & $\mathrm{R}$ & $\mathrm{R}$ & 24 & I & $\mathrm{R}$ \\
\hline Ticarcillin & 32 & $-*$ & $\mathrm{R}$ & $>256$ & $-*$ & $\mathrm{R}$ \\
\hline Temocillin & 24 & $-*$ & $\mathrm{R}$ & 24 & $-*$ & $\mathrm{R}$ \\
\hline Piperacillin & 24 & I & $\mathrm{R}$ & 12 & $\mathrm{~S}$ & I \\
\hline Piperacillin $+\mathrm{TAZ}^{b}$ & 4 & $\mathrm{~S}$ & $\mathrm{~S}$ & 2 & $\mathrm{~S}$ & $\mathrm{~S}$ \\
\hline
\end{tabular}




\begin{tabular}{|c|c|c|c|c|c|c|}
\hline Cefoxitin & $>256$ & $\mathrm{R}$ & $\mathrm{R}$ & 16 & I & I \\
\hline Cefuroxime & 24 & I & $\mathrm{R}$ & $>256$ & $\mathrm{R}$ & $\mathrm{R}$ \\
\hline Cefotaxime & 8 & $\mathrm{R}$ & $\mathrm{R}$ & $>32$ & $\mathrm{R}$ & $\mathrm{R}$ \\
\hline Ceftazidime & 24 & $\mathrm{R}$ & $\mathrm{R}$ & 24 & $\mathrm{R}$ & $\mathrm{R}$ \\
\hline Ceftazidime $+\mathrm{AVI}^{c}$ & 0.38 & $\mathrm{~S}$ & $\mathrm{~S}$ & 0.75 & $\mathrm{~S}$ & $\mathrm{~S}$ \\
\hline Cefepime & 0.125 & $\mathrm{~S}$ & $\mathrm{~S}$ & 0.094 & $\mathrm{~S}$ & $\mathrm{~S}$ \\
\hline Ceftolozane $+\mathrm{TAZ}^{b}$ & 0.5 & $\mathrm{~S}$ & $\mathrm{~S}$ & 2.5 & I & $\mathrm{R}$ \\
\hline Imipenem & 0.25 & $\mathrm{~S}$ & $\mathrm{~S}$ & 0.19 & $\mathrm{~S}$ & $\mathrm{~S}$ \\
\hline Meropenem & 0.032 & $\mathrm{~S}$ & $\mathrm{~S}$ & 0.023 & $\mathrm{~S}$ & $\mathrm{~S}$ \\
\hline Ertapenem & 0.012 & $\mathrm{~S}$ & $\mathrm{~S}$ & 0.012 & $\mathrm{~S}$ & $\mathrm{~S}$ \\
\hline Aztreonam & 2 & $\mathrm{~S}$ & I & 3 & $\mathrm{~S}$ & I \\
\hline
\end{tabular}

S: susceptible; I: intermediate; R: resistant

* values not available

${ }^{a}$ CLA: clavulanic acid

${ }^{b}$ TAZ: tazobactam

${ }^{c}$ AVI: avibactam

\section{Genetic support of bla ${ }_{\mathrm{CMY}-136}$ gene}

Kieser $^{7}$ extracted DNA from E. coli EC13 was electroporated into electrocompetent E. coli TOP10 cells and plated on ampicillin-containing plates $(100 \mathrm{mg} / \mathrm{L})$. Several colonies that grew on ampicillin were confirmed by PCR and sequencing to be blaCMY-136 positive. Kieser DNA of $E$. coli $\mathrm{EC} 13$ and of electrotransformed E. coli TOP10 cells run on a $0.7 \%$ agarose gel revealed a single plasmid, pCMY-136 of ca. $90 \mathrm{Kbp}$ in both isolates. Whole genome sequencing using Illumina technology of E. coli TOP10 (pCMY-136) revealed a 90844 bp plasmid in size, that belonged to the IncI1 group, and carried a single antibiotic resistance gene, bla $a_{\mathrm{CMY}-136}$. BLAST search showed the closest deposited plasmid sequence to be the bla $a_{\mathrm{CMY}-2}$ carrying plasmid pCVM22462 (accession number $\underline{\mathrm{CP} 009566.1})^{8}$, isolated from a Salmonella enterica strain. It is 99.98\% identical to pCMY-136, excluding a 3,888-bp fragment that is absent in pCMY-136. This deletion is located 1,965-bp upstream of bla $a_{\mathrm{CMY}-136} /$ bla $_{\mathrm{CMY}-2}$ genes. This 3,888-bp fragment contains 3 hypothetical protein ORFs and occurred contiguously downstream of an IS1294-like insertion sequence present on both plasmids.

\section{Purification and biochemical characterization of CMY-136}


CMY-136 and CMY-2 were overexpressed and purified by Immobilized Metal Affinity Chromatography (IMAC), and purity of both enzymes was confirmed by SDS-PAGE, where a single band of ca. $41 \mathrm{kDa}$ was observed. Steady-state kinetic parameters were determined to compare the catalytic activity of CMY-136 to that of CMY-2 against several $\beta$-lactams (Table 2). CMY-136 showed around a 10-fold increase in $\mathrm{Km}$ for most $\beta$-lactams assayed, with however, a few exceptions: (i) cephalothin (CEF), which showed a slightly higher affinity for CMY-136 than CMY-2, although other authors have reported a lower Km for CMY-2 against $\mathrm{CEF}^{9,10,11}$; (ii) cefoxitin (FOX), which showed a practically identical Km for both enzymes; (iii) $\beta$-lactams with a very high affinity for CMY-2 and a bulky, non-linear, R1 substituent, namely cefuroxime (CXM), cefotaxime (CTX) and ceftazidime (CAZ), which show a $10^{3}-10^{4}$-fold increase in $\mathrm{Km}$ for CMY136; (iv) other $\beta$-lactams, namely ticarcillin (TIC) and cloxacillin (CLX), for which CMY-2 shows high affinity and no hydrolysis unlike CMY-136 that does hydrolyze them (see Fig. S2 for the meaning of the R1 and R2 substituents).

CMY-136 showed a decreased $k_{\text {cat }}$ for most of the preferred substrates of CMY-2, namely amino-penicillins and first generation cephalosporins, and also cefoxitin. However, it also shows an increased $k_{\text {cat }}$ for cefepime and ceftolozane, and a remarkably increased $k_{\text {cat }}\left(10^{2}-10^{3}\right.$-fold) for those $\beta$-lactams for which the most dramatic $\mathrm{Km}$ increase was also seen, CAZ, CTX, CXM. CMY-136 is also capable of hydrolysing cloxacillin and ticarcillin, which are not hydrolysed by CMY-2. In terms of their catalytic efficiency, all these translate into CMY-136 showing a lower efficiency than CMY-2 for most $\beta$-lactams tested, except for those not hydrolysed by CMY-2, TIC and CLX, and also ceftolozane.

Table 2 Steady-state kinetic parameters of $\beta$-lactamases CMY-136 and CMY-2.

\begin{tabular}{lcclccc}
\hline \multirow{2}{*}{ Substrate } & \multicolumn{2}{c}{$K_{\mathrm{m}}(\mu \mathrm{M})$} & \multicolumn{2}{c}{$k_{\text {cat }}\left(\mathrm{s}^{-1}\right)$} & \multicolumn{2}{c}{$k_{\text {cat }} / K_{\mathrm{m}}\left(\mathrm{mM}^{-1} \mathrm{~s}^{-1}\right)$} \\
\cline { 2 - 7 } & CMY-2 & CMY-136 & CMY-2 & CMY-136 & CMY-2 & CMY-136 \\
\hline Benzylpenicillin & $0.6^{b}$ & $10.1^{*}$ & $24^{b}$ & 7.83 & $40000^{b}$ & 775 \\
Cloxacillin & $0.0002^{*}$ & 543 & N.H. & 0.47 & N.H. & 0.9 \\
Ampicillin & $0.16^{c}$ & $3.5^{*}$ & $0.55^{c}$ & 0.43 & $3437^{c}$ & 122 \\
& & \multicolumn{2}{c}{6} & & &
\end{tabular}




$\begin{array}{lcccccc}\text { Ticarcillin } & 0.03^{*} & 1.7^{*} & \text { N.H. } & 0.15 & \text { N.H. } & 86 \\ \text { Cephaloridine } & 130^{a} & 1532 & 421^{a} & 169 & 3240^{a} & 110 \\ \text { Cephalothin } & 90^{a} & 35 & 217^{a} & 21.65 & 2400^{a} & 618 \\ \text { Cefoxitin } & 0.15^{b} & 0.2^{*} & 0.35^{b} & 0.02 & 2333^{b} & 111 \\ \text { Cefuroxime } & 0.005^{b} & 21.6 & 0.014^{b} & 9.42 & 2800^{b} & 436 \\ \text { Cefotaxime } & 0.001^{a} & 20 & 0.007^{a} & 4.71 & 7000^{a} & 236 \\ \text { Ceftazidime } & 0.15^{a} & 2360 & 0.005^{a} & 6.26 & 33^{a} & 2.7 \\ \text { Cefepime } & 412 & 3588 & 0.37 & 1.79 & 0.9 & 0.5 \\ \text { Ceftolozane } & 200 & 1587 & 0.69 & 13.27 & 3.5 & 8.4 \\ \text { Imipenem } & 0.8^{a} & 10.9 & 0.03^{a} & 0.01 & 38^{a} & 1.2 \\ \text { Aztreonam } & \text { N.H. }{ }^{b} & \text { N.H. } & \text { N.H. }{ }^{b} & \text { N.H. } & \text { N.H. } & \text { N.H. }\end{array}$

Errors for determined kinetic values are below $10 \%$

N.H.: no hydrolysis detected with concentrations of substrate and enzymes up to $1,000 \mathrm{mM}$ and 400 $\mathrm{nM}$, respectively.

${ }^{a}$ taken from ${ }^{12,5}$

${ }^{b}$ taken from ${ }^{9}$

${ }^{c}$ taken from ${ }^{11}$

*Km values determined by inhibition assays as explained in experimental procedures

Table 3 shows $\mathrm{IC}_{50}$ values for aztreonam, tazobactam, and clavulanic acid. CMY-136 is similarly susceptible to inhibition by tazobactam as CMY $-2^{13,14}$.

Table $3 \mathrm{IC}_{50}$ values $(\mu \mathrm{M})$ for inhibitors of CMY-136 and CMY-2.

\begin{tabular}{lcc}
\hline \multicolumn{1}{c}{ Inhibitor } & CMY-2 & CMY-136 \\
\hline Tazobactam & $3.2^{\mathrm{a}}$ & 5.5 \\
Clavulanic acid & N.D. & 137 \\
Aztreonam & N.D. & 0.11 \\
\hline
\end{tabular}

N.D.: not determined

${ }^{a}$ taken from ${ }^{13}$

\section{CMY-136 crystallization and X-ray crystallography}

CMY-136 crystallized in 0.1 M HEPES pH 7.5; 20\% w/v PEG4000; 10\% isopropanol. The structure was determined by molecular replacement using the deposited CMY-2 structure (PDB: 1ZC2) as a template, and the model was refined to $1.60 \AA$ (Table 4). R/Rfree values seem to be relatively high for the resolution obtained, which may be a consequence of merging two datasets (see experimental procedures). The asymmetric unit contains two protein chains, A and B, showing 
no significant conformational differences between them. They have been modelled with 359 residues each, with clear electron density observed for all regions of the protein. Several ordered water molecules have been modelled in the structure as well as phosphate anions and isopropyl alcohol molecules, from the crystallization condition. Both chains present the typical class $\mathrm{C}$ fold with an $\alpha$-helical region and a mixed $\alpha$-helix/ $\beta$-sheet region. $99 \%$ of all residues are inside the favoured regions of the Ramachandran plot, and $1 \%$ in the allowed regions.

Table 4 Crystallography data collection and refinement statistics.

\begin{tabular}{ll}
\hline Data collection & \\
\hline Space group & $\mathrm{P} 1211$ \\
Cell dimensions & \\
a, b, c $(\AA)$ & $60.58,58.09,100.08$ \\
$\quad \alpha, \beta, \gamma\left({ }^{\circ}\right)$ & $90.00,89.97,90.00$ \\
Resolution $(\AA)$ & $16.1-1.60(1.62-1.60)$ \\
Rmerge & $0.092(0.67)$ \\
I/ $\sigma(\mathrm{I})$ & $9.9(3.9)$ \\
Completeness $(\%)$ & $95.0(96.3)$ \\
Redundancy & $4.2(4.4)$ \\
CC $(1 / 2)$ & $0.991(0.775)$ \\
& \\
\hline Refinement & \\
Resolution range $(\AA)$ & $16.10-1.60$ \\
No. unique reflections & 87,503 \\
Rwork/Rfree & $23.4 \% / 26.4 \%$ \\
No. non-hydrogen atoms & \\
Protein & 5,642 \\
Water & 396 \\
Ligand/Ions & 67 \\
Total & 6105 \\
Average B, all atoms $(\AA 2)$ & 19.8 \\
Protein & 19.4 \\
Water & 25.2 \\
Ligand/Ions & 29.6 \\
Root mean squared deviations & \\
Bond lengths $(\AA)$ & 0.01 \\
Bond angles $\left({ }^{\circ}\right)$ & 1.04 \\
\hline Valn in & \\
\hline & \\
\hline
\end{tabular}

Values in parenthesis correspond to the higher resolution bin. 
CMY-136 shows the same overall conformation as CMY-2, and 3D alignment reveals a C $\alpha$ RMSD of 0.465-0.486 ̊ between them. Several structural differences can be found when comparing CMY136 (Fig. 1) to CMY-2: (i) A phosphate molecule is positioned inside the active site of both CMY136 chains, with one of its oxygens buried inside the oxyanion hole. CMY-2 was crystallized with a citrate molecule inside the active site; (ii) The conformation adopted by the TYR221 in CMY-2, which interacts with the backbone of the $\Omega$-loop at SER212 O and GLN215 O via two bridging waters, is different from the conformation found in CMY-136 for HIS221, whose sidechain invades the active site cavity, hydrogen bonding the aforementioned phosphate oxygen atom. The TYR221 residue and its conformation are highly conserved in all class $\mathrm{C}$ enzymes reported to date (with the exception of PDC-85) and in all class C crystallographic structures deposited in the PDB that have a consensus length $\Omega$-loop; (iii) In turn, the sidechain of ASP217 turns towards the interior of the $\Omega$ loop to partially occupy the space left by the conformational change of the Y221H mutation; (iv) The conformation adopted by the backbone of the $\Omega$-loop residues ARG204 to GLN215 in CMY136 is different from the one found in CMY-2, that is also highly conserved in deposited class C structures with a consensus length loop, which translates into a backbone atoms RMSD between said residues in both crystal structures of $2.176-2.219 \AA$, a $4.56-4.68 \AA$ displacement in the position of the C $\alpha$ of VAL211, and the loss of the hydrogen bonds between GLU61 O $\varepsilon$ and VAL211 N (Fig. 2) and between VAL209 O and GLY202 N (Fig. 2B). Two water molecules, w502 and w504,occupy the usual space of residues V209-V211 backbone, and partially replace those lost hydrogen bonds (Fig. 2B); (v)The configuration of HIS210 sidechain is shifted from being positioned between, and interacting via pi-pi stacking with, TYR199 and TRP201 in CMY-2 another highly conserved feature of class C structures, that fill this space with either HIS210 or ARG210 sidechains - to not directly interacting with any other residue, and hydrogen bonding w502 (Fig. 2B). The space between TYR199 and TRP201 in the CMY-136 structure is filled by LYS290 from another CMY-136 chain in the neighbouring asymmetric unit in the crystal structure; (vi) The conformation of the active site cavity residues and the hydrogen bond network between them is 
highly conserved, with the exception of TYR150, which is slightly shifted towards the phosphate anchored in the active site, with a $3.1 \AA$ displacement of its hydroxyl oxygen atom compared to TYR150 of the CMY-2 structure (Fig. 1 and Fig. S3). A water molecule can be found occupying this position, and TYR150 is still hydrogen bonding LYS67, and now also hydrogen bonds with the phosphate molecule and possibly ASN152, but no longer interacts with LYS315.

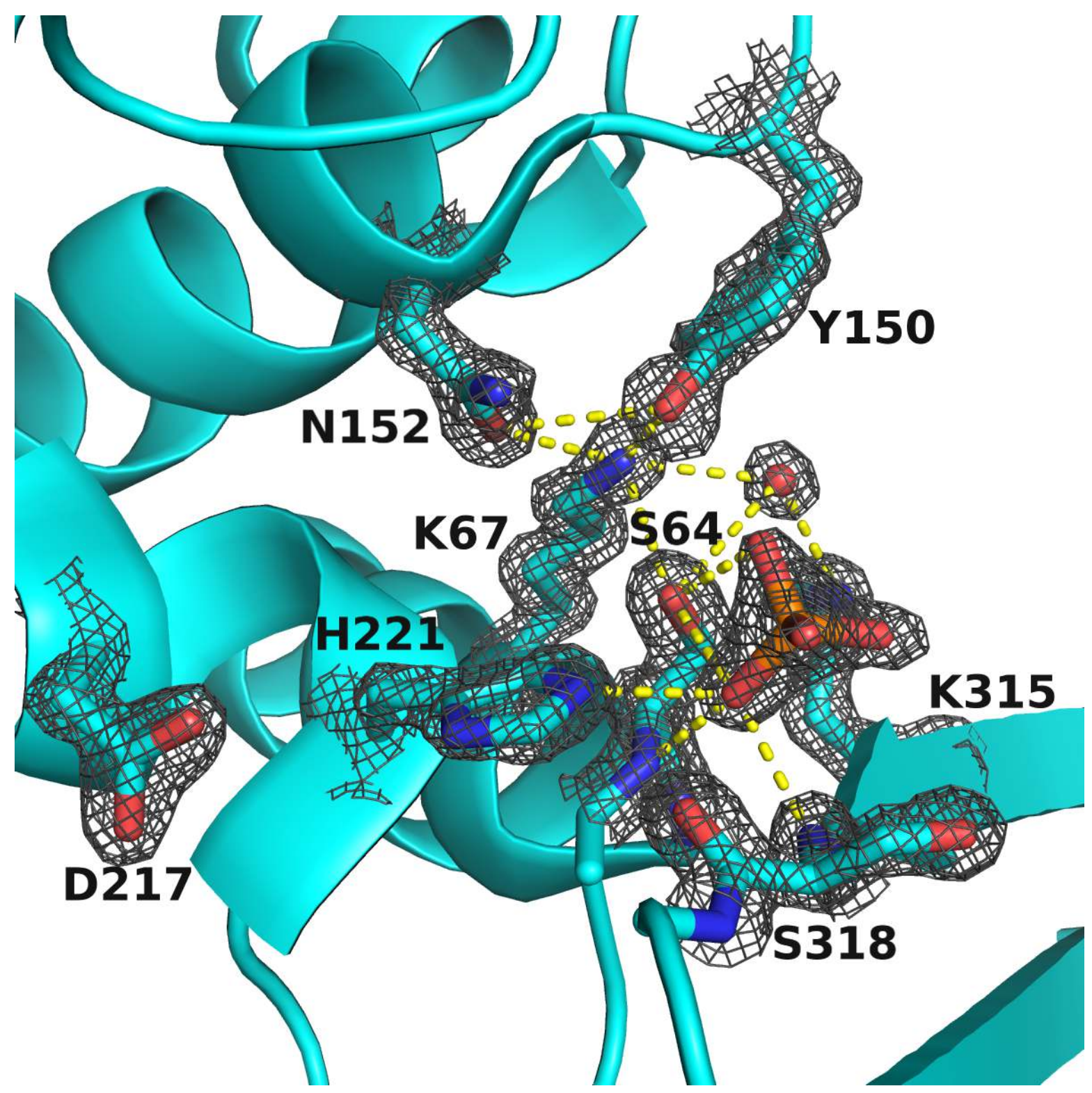

Fig. 1 Crystal structure of CMY-136. Active site cavity of CMY-136, with a phosphate molecule interacting with the oxyanion hole and other important residues. Note the conformation of the H221 
sidechain, the position shift of Y150, and the water molecule occupying its hydroxyl usual position. Hydrogen bonds are depicted as dashed yellow lines. 2Fo-Fc electron density (contoured at $2 \sigma$ ) is shown.

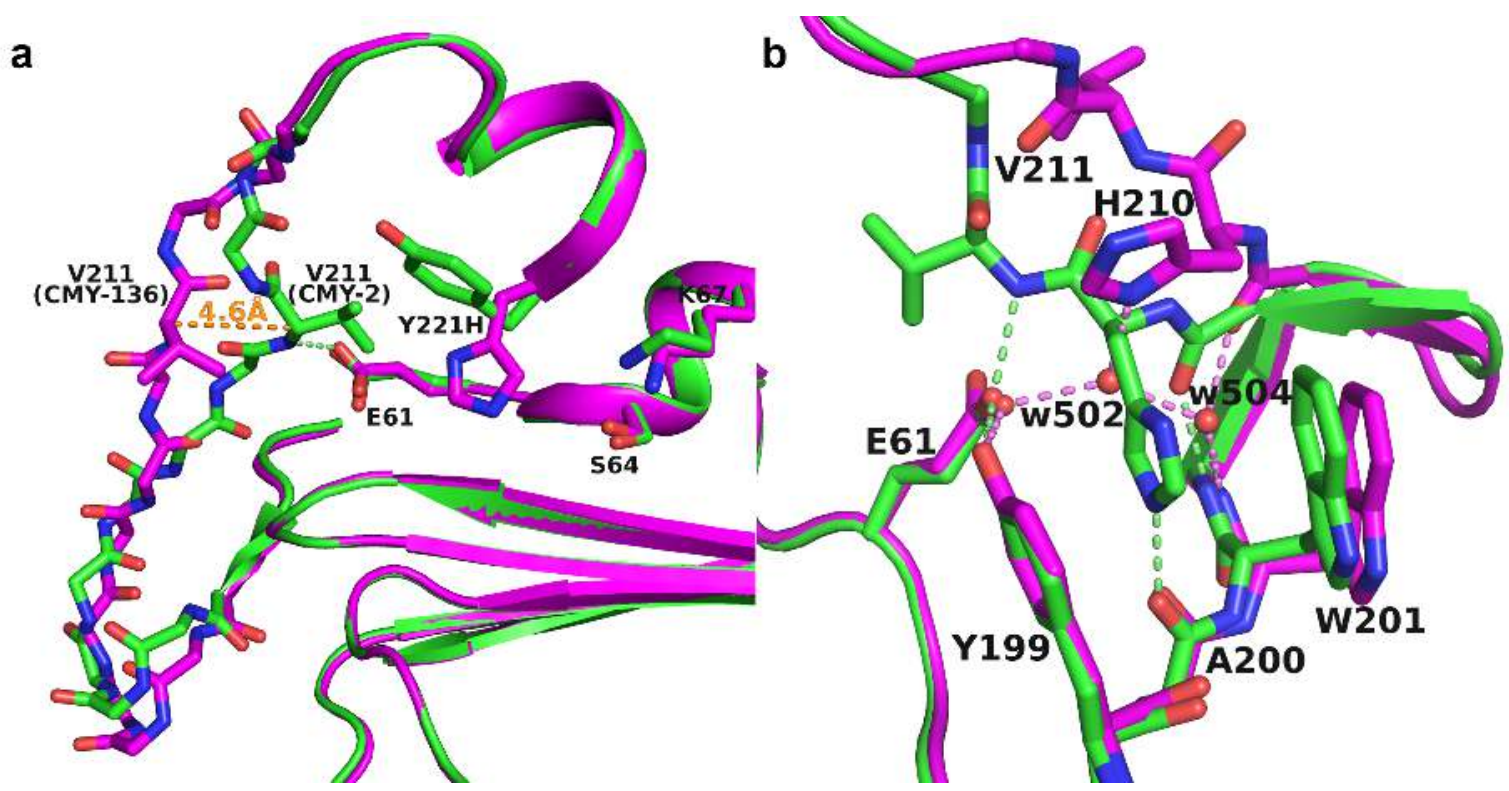

Fig. 2 Comparison of the $\Omega$-loop of CMY-136 and CMY-2. CMY-2 and CMY-136 are shown in green and magenta, respectively. a) Front view, the conformational change in the $\Omega$-loop for CMY136 widens the active site cavity, displacing V211 C $\alpha$ about $4.6 \AA$, and breaking the hydrogen bond with E61. Hydrogen bonds are depicted as dashed yellow lines. Displacement depicted as dashed orange lines. b) Side view, the conformational change also displaces H210 sidechain from its usual position interacting with residues 199-201. Hydrogen bonds are displayed as colored dashed lines according to model colors.

\section{Modelling of covalent complexes of CMY-2 and CMY-136 with $\beta$-lactams}

As no crystal structure of CMY-2 co-crystallized with $\beta$-lactams is available, covalent complexes of CMY-2 and CMY-136 with ceftazidime, cefotaxime and cefuroxime were created by superposing the structures of CMY-2 and CMY-136 with the available structures of class C enzymes with 
ceftazidime (PDB code 1IEL) and cefotaxime (PDB code 4KG2), and in the case of cefuroxime, the covalently bound substrate was modelled on the conformation of the cefotaxime complex, given no covalent complex of class $\mathrm{C}$ enzymes with cefuroxime is available and both molecules show minor differences. Results show that in the case of CMY-2, acyl-enzyme complexes have minor clashes with L293 (Fig. 3A). In the case of CMY-136 numerous overlaps with residues H221 and Y150 exist (Fig. 3C), which may explain the approximately 1000-fold increase in $\mathrm{Km}$ values for expanded spectrum cephalosporins for CMY-136 compared to CMY-2. Covalent complexes were then subjected to energy minimization steps, and results showed the conformational changes needed to resolve overlaps in the case of CMY-2 were smaller than those for CMY-136 (Fig. 3B and D, respectively). Both $\mathrm{H} 221$ and the $\mathrm{R} 1$ substituent of cephalosporins are displaced in opposite directions in the CMY-136 complexes, pushing the substrate outwards from the active site cavity and H221 towards the $\Omega$-loop. In the case of CMY-2, displacements were smaller in magnitude, and they tended to approach the R1 substituent towards Y221 (see Fig. S2 for the meaning of the R1 substituent). 


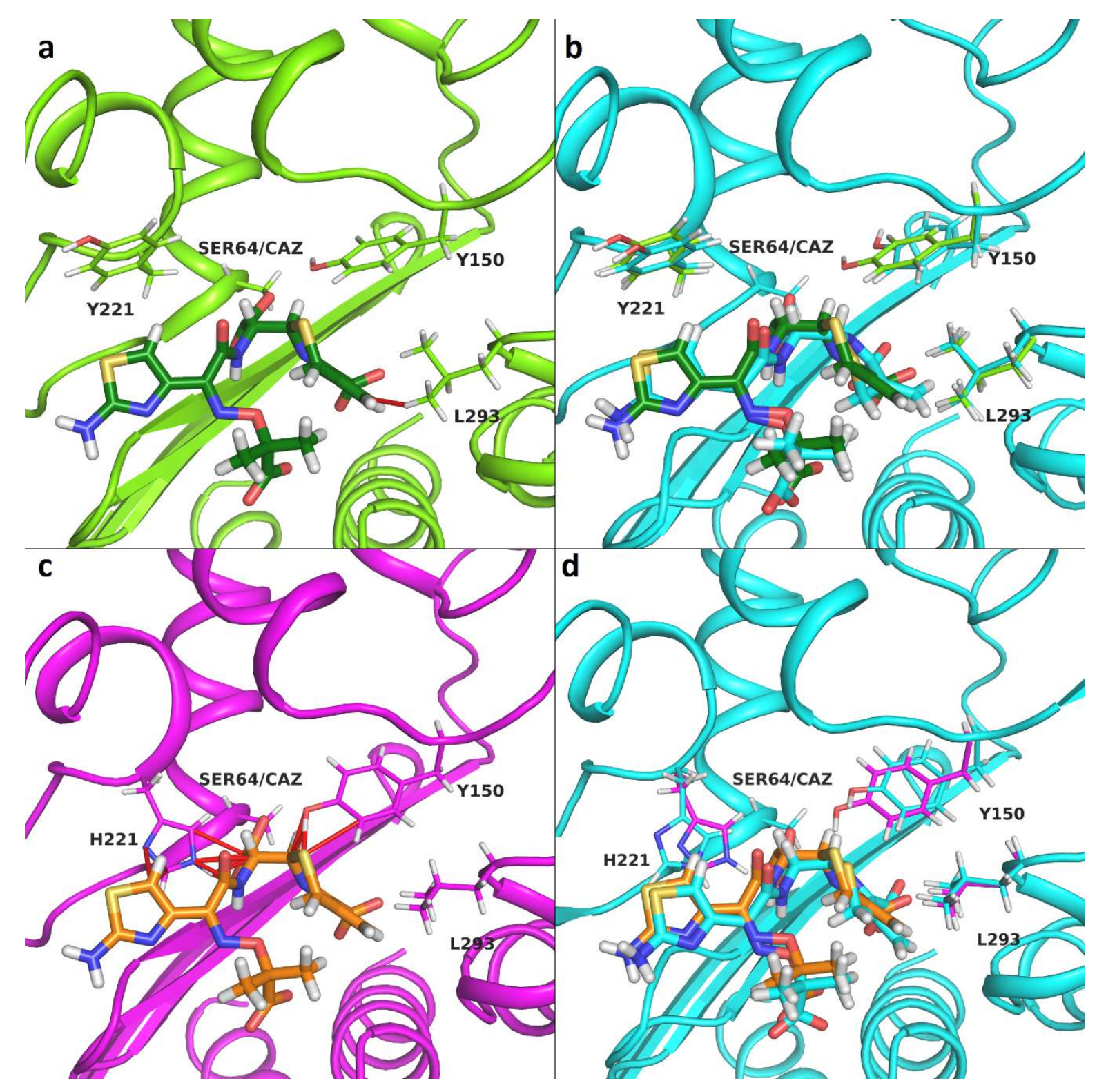

Fig. 3. Modelling of covalent complexes of CMY-2 and CMY-136 with ceftazidime. Covalent complexes were made by superposing the structures of CMY-2 or CMY-136 on the covalent complex of ceftazidime with AmpC (PDB code 1IEL). These were subjected to energy minimization. Covalent complex of CMY-2 with ceftazidime before (a) and after (b) minimization, as well as covalent complex of CMY-136 with ceftazidime before (c) and after (d) minimization are shown. Only relevant residues from CMY enzymes and ceftazidime are shown as sticks for simplicity. Overlaps between the ligand and the protein are depicted as red lines. In figures $\mathbf{b}$ and $\mathbf{d}$, 
both the minimized (cyan) and initial (green for CMY-2, orange for CMY-136) structures are shown for comparison.

\section{Molecular dynamics simulations of CMY-2 and CMY-136}

To investigate the role of the Y221 mutation on the dynamics of the protein structure, $10 \mathrm{~ns}$ molecular dynamics (MD) simulations were performed in triplicate with four different systems (Fig. S4): CMY-2, CMY-136, CMY-136 with the $\Omega$-loop modelled on the CMY-2 structure, and CMY-2 with the $\Omega$-loop modelled on the CMY-136 structure. For these MD simulations, citrate and phosphate ions were removed from the active sites of CMY-2 and CMY-136 structures, respectively. Root-mean-square deviation (RMSD) analysis of the trajectories reveals that the simulations reached stability within $2 \mathrm{~ns}$ and remained stable after that. Root-mean-square fluctuation (RMSF) analysis of $\mathrm{C} \alpha$ atoms in CMY-2 and CMY-136, expressed as B-factors, reveals some differences between them, regarding the flexibility of several parts of the structure (Fig. 4A). CMY-136 displays increased flexibility in the $\Omega$-loop and H7 helix, from R204 up to H221, except for a short segment in the middle. An increased flexibility can also be found for part of the Q120 loop, part of H10 and H11 helixes, and the C terminus of B3 strand, S318 and T319. Other loops around CMY-136 also have increased flexibility compared to CMY-2, to a lesser extent. There are also segments of decreased flexibility towards the exterior of the structure, contiguous to the previously described regions, the N-terminus of $\mathrm{H} 10$ helix and its preceding loop, C-terminus of H11 helix, H4 helix, G320, part of helixes H5 and H6, directly behind H4 helix in the tertiary structure, and also S343, next to the C-terminus of strand B3. It should be noted that R349, in the middle of H11, also shows decreased flexibility. Regions with increased flexibility can be found surrounding and forming the walls of the active site cavity, with regions of decreased flexibility further away (Fig. 4B). 
a

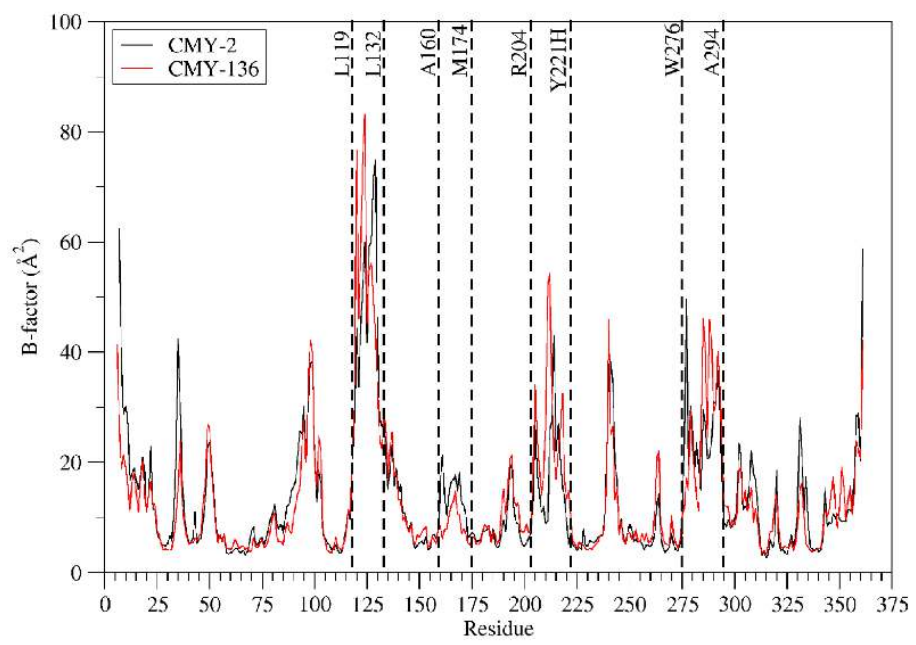

b

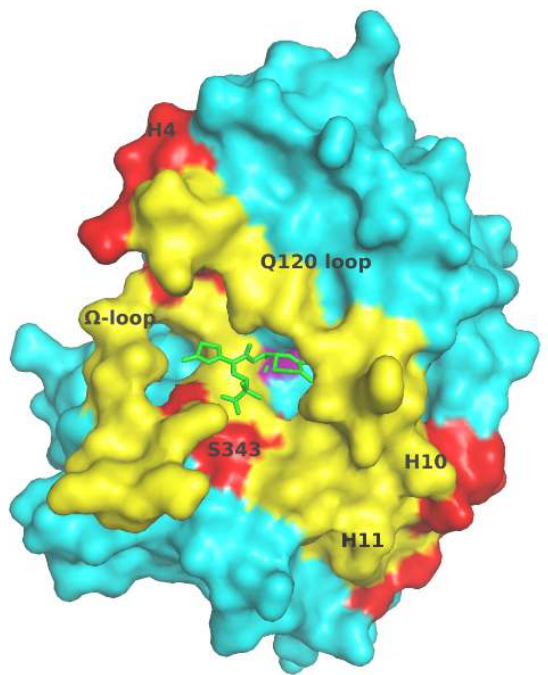

Fig. 4. Altered flexibility of CMY-136 Structure. a) CMY-2 and CMY-136 RMSF analysis, expressed as $\mathrm{C} \alpha$ B-factors. Compared to $\mathrm{CMY}-2$, several regions displayed increased or decreased flexibility in the CMY-136 structure during MD simulation: part of the $\Omega$-loop (R204-Y221), Q120 loop (P118-R126), C-terminus of B3 strand (S318-G320), helixes H4 (D127-L131), H5 (A160G167), H6 (S169-M174), H10 (P277-A295), H11 (S343-Q361). b) Surface representation of CMY136 in the acyl-enzyme complex with CAZ, which is represented as green sticks. The surface of S64 is colored in purple. The surface of regions with increased flexibility, determined by C $\alpha$ Bfactors, are colored in yellow, and those with decreased flexibility in red.

The MD simulations also demonstrate how the sidechain of Y221 in CMY-2 remains constantly in the same conformation, pointing outwards of S64, with its hydroxyl hydrogen bonding with the backbone of the $\Omega$-loop directly or through bridging waters (Fig. 5A). In contrast, H221 in CMY136 displays much more flexibility, adopting several conformations in the active site throughout the MD simulation (Fig. 5B and 6). Other parts of the $\Omega$-loop also show increased sidechain flexibility (Fig. 6), namely H210-S212 and Y199-W201, which normally interact closely with each other and with E61. 

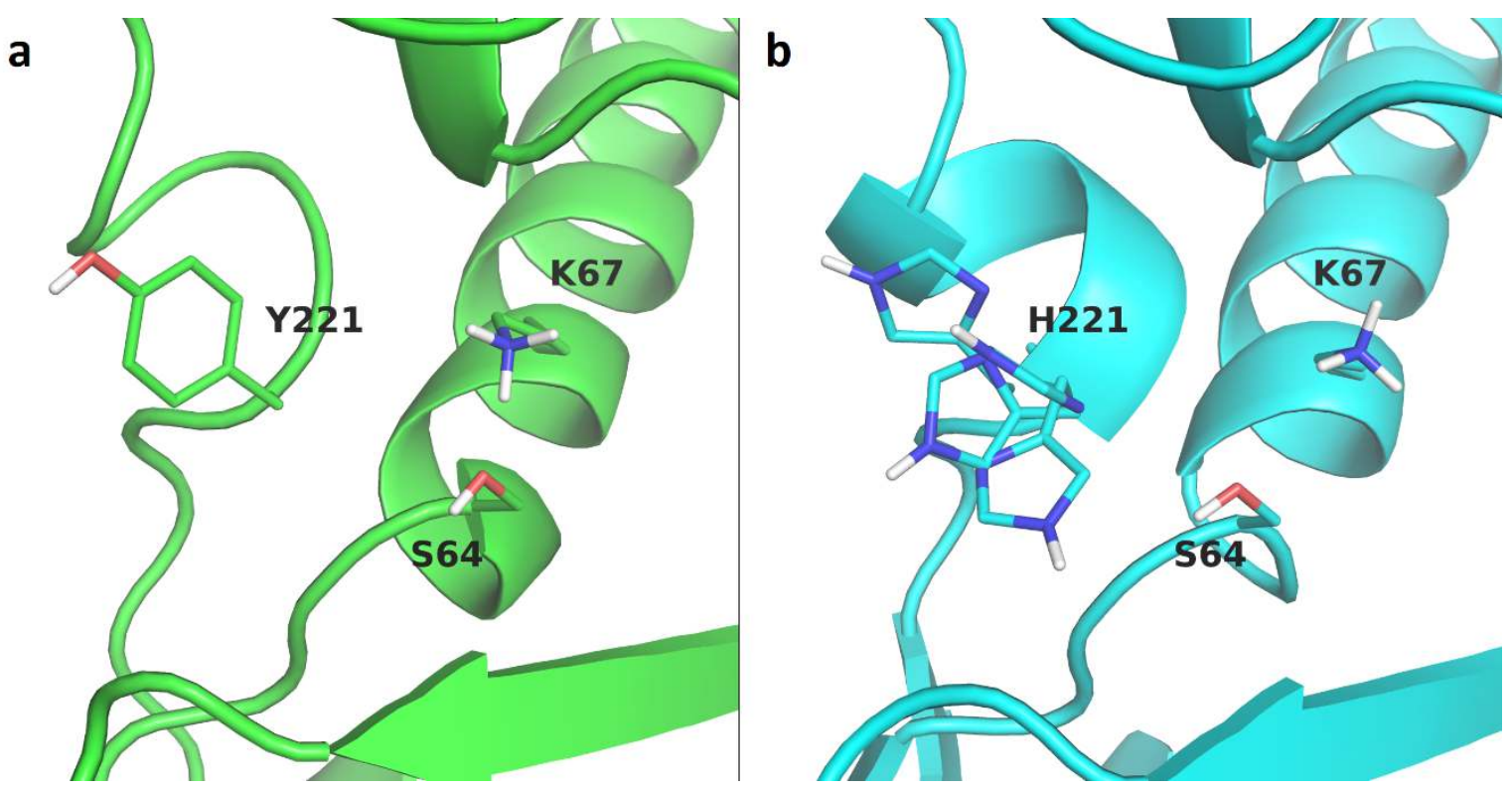

Fig. 5. Flexibility of TYR 221 in CMY-2 and HIS 221 in CMY-136. A) Snapshot from the CMY2 MD simulation depicting the only conformation of Y221 observed throughout the $10 \mathrm{~ns}$ simulation. B) H221 conformations observed in four representative snapshots of the CMY-136 MD simulation. One of the conformations adopted is similar to that of Y221 for CMY-2 seen in panel A. 


\section{All Atoms Protein B-factor $\left(\AA^{2}\right)$}

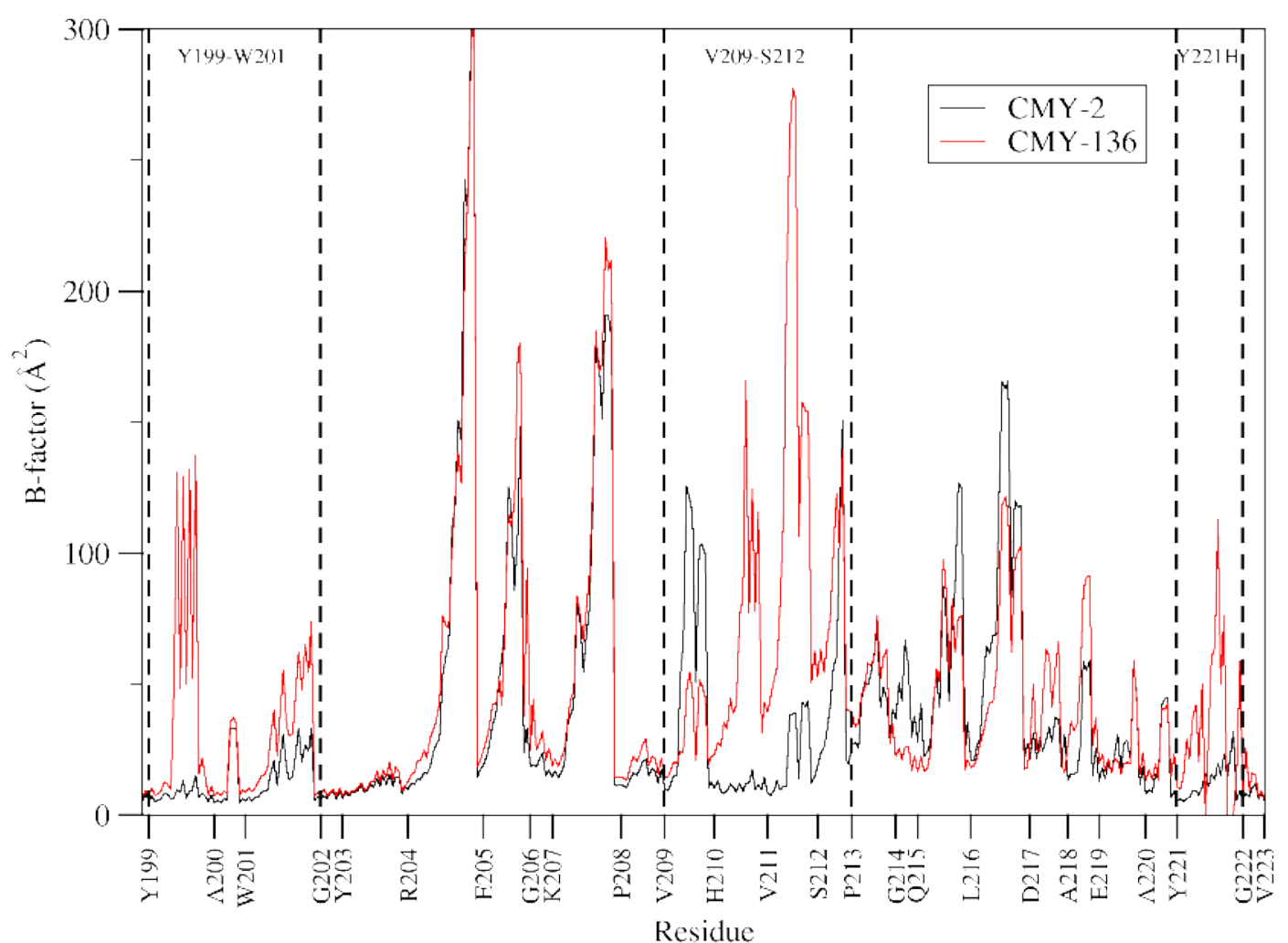

Fig. 6. Flexibility of $\Omega$-loop in CMY-136 vs CMY-2. Individual atom B-factors of CMY-136 and CMY-2 $\Omega$-loops. Note the increased flexibility of residue 221 in CMY-136, and also of regions H210-S212 and Y199-W201, which interact in CMY-2 as shown in Fig. 2.

This same H221 flexibility can be observed for the simulation of the CMY-136 model with the $\Omega$ loop, $\mathrm{H} 7$ and $\mathrm{H} 221$ sidechain starting with the same conformation as the CMY-2 crystal structure. In contrast, for the CMY-2 model with the $\Omega$-loop, H7 and Y221 sidechain starting with the same conformation as the CMY-136 crystal structure, the native conformation of Y221 is not reached. Simulations for both enzymes starting either with the $\Omega$-loop in the conformation observed in their own crystal structure, or in that of the other enzyme, seem to show that the conformation acquired by the $\Omega$-loop during the simulations is independent of starting conformation. Snapshots of these four simulations were extracted every $1 \mathrm{~ns}$ and fitted on the CMY-2 or CMY-136 crystal structure 
as references. $\Omega$-loop C $\alpha$ RMSD between the snapshot and the reference was determined, to assess conformational differences (Fig. S5). The analysis shows that both CMY-136 simulations, starting with a $\Omega$-loop, H7 and H221 conformation as that observed in either the CMY-136 crystal structure or the CMY-2 crystal structure, present a lower RMSD compared to CMY-136 than to CMY-2 crystal structures towards the end of the simulation. Similarly, for the CMY-2 simulation starting from the CMY-2 crystal structure conformation, some fluctuations can be observed during the simulation but finally the RMSD is also lower for its own native crystal structure than for that of the other enzyme, and in the same magnitudes as observed for CMY-136. In contrast, in the simulation of CMY-2 starting with the $\Omega$-loop, H7 and Y221 conformation of the CMY-136 loop does not converge to the CMY-2 crystal structure conformation and remains closer to the CMY-136 crystal structure conformation. As mentioned earlier, for this simulation, Y221 does not acquire the native conformation observed in all class $\mathrm{C}$ structures.

\section{DISCUSSION}

We report here a novel $\beta$-lactamase belonging to the CMY-2 family, with an unusual mutation, Y221H, in a highly conserved position in class C $\beta$-lactamases. Analysis of the plasmid harbouring bla $a_{\mathrm{CMY}-136}$ suggests it may have originated by mutation deriving from the bla $a_{\mathrm{CMY}-2}$ harbouring plasmid pCVM22462. It is noteworthy that the 3888bp fragment absent from pCMY136 but present in pCMY-2 occurs directly adjacent to IS1294, which may suggest its involvement in the deletion of this fragment ${ }^{15}$.

The biochemical characterization of CMY-136 suggests it to be less efficient than CMY-2 in hydrolysing most $\beta$-lactams, the exception being ceftolozane, and also those not hydrolysed by the latter, cloxacillin and ticarcillin. The results for these three $\beta$-lactams corroborate what can be observed in the MIC assays. However, other significant differences in MICs for CMY-136 when compared to CMY-2 (increased MIC for cefotaxime and cefuroxime, and decreased MIC for cefoxitin) are not reflected in the catalytic efficiencies determined. In this regard, it has been 
previously suggested that for class $\mathrm{C}$ enzymes, $k_{\text {cat }}$ may better represent the degree of resistance towards $\beta$-lactams ${ }^{16,17}$. Therefore, the comparison of $k_{\text {cat }}$ may explain better what is observed for the MICs. A 10-fold increase in $k_{\text {cat }}$, or bigger, is observed for CMY-136 for cloxacillin, ticarcillin, cefuroxime, cefotaxime, ceftazidime, and ceftolozane, and a 10-fold decrease for cephalothin and cefoxitin. This corresponds well with observed MIC results, except for cloxacillin and cephalothin, for which MICs were not determined, and ceftazidime, which showed no difference in MIC between CMY-136 and CMY-2.

These results for CMY-136 are consistent with previously published results for similar cases. PDC-85, a variant of the chromosomally-encoded AmpC of Pseudomonas aeruginosa, is the only other class $\mathrm{C}$ enzyme presenting a Y221H mutation ${ }^{18}$. Similarly to CMY-136, the PDC-85 variant showed an increase in MIC for ticarcillin and ceftolozane/tazobactam, and a decreased susceptibility to inhibition by cloxacillin, probably related to CMY-136 being capable of hydrolysing cloxacillin. In a different study, it was observed that the E. coli AmpC Y221G laboratory mutant showed an increased $K_{m}$ and $k_{c a t}$ for cefotaxime, while at the same time significantly losing activity on cephalothin ${ }^{17}$. The authors evidenced that this mutation decreases the enzyme thermal stability, increase flexibility, and expand the active site cavity, and attribute the increase in cefotaxime hydrolysis to these effects, allowing for a better accommodation of the acylenzyme complex into a catalytically competent conformation for de-acylation. For cephalothin, they propose the now missing stabilizing interactions between the substrate and the Y221G mutation to be responsible for the loss of hydrolysis.

The crystal structure of CMY-136 shows the same conformation as that of CMY-2, except for those differences described in the Results section. Just as for the Y221G AmpC mutant study, in CMY-136 the space no longer occupied by Y221 is partially occupied by D217, which turns towards the active site cavity. Another possible common feature is the expansion of the active site cavity, as the CMY-136 $\Omega$-loop conformation displaces the V211 C $\alpha$ from its usual position in class C structures by about $4.6 \AA$. It is unclear whether the changes observed in the CMY-136 
conformation are the result of the crystal configuration and interactions between neighbouring chains in it, the crystallization with a phosphate molecule in the active site, the consequences of the Y221H mutation, or a combination of them.

In order to investigate the high differences in affinity for expanded spectrum cephalosporins between CMY-2 and CMY-136, covalent complexes were created by superimposing the structures with available complexes and subjected to energy minimization, to evidence the conformations adopted by the acyl-enzyme complexes. As shown in Fig. 3, minimization does not lead to important conformational changes in the case of CMY-2. Superposition of the CMY-136 crystal structure on the complexes structures shows that there would be significant overlapping between the R1 sidechain of $\beta$-lactams and the H221 sidechain. This serves to explain why CMY-136 may show an increased $K m$ for most $\beta$-lactams: in order for the $\beta$-lactam and the enzyme to interact, either the R1 sidechain or the H221 sidechain, or both, must adopt a different conformation from the one seen in most structures of class C enzymes in complex with substrates or the CMY-136 structure, respectively. Such conformational change most likely implies an energetic cost, that in turn represents an increase in $\mathrm{Km}$. Furthermore, this higher energetic cost - and increase in $\mathrm{Km}$ - for CMY-136 interacting with most $\beta$-lactams seems to correlate with how big, bulky, ramified or rigid is the R1 substituent. There are much larger increases in $K m$ for cloxacillin, ceftazidime, cefuroxime and cefotaxime than for those with a simpler R1 sidechain like benzylpenicillin or cephalothin. One possible explanation as for why such $\mathrm{Km}$ increase is less intense or doesn't exist for CEF or FOX compared to CAZ or CXM, is that the R1 sidechain of the former is not as bulky, ramified, or rigid as for the others, and thus they may prove easier to accommodate inside the active site of CMY-136 with its Y221H mutation, implying less movement restrain for H221, or making more favourable contacts and hydrogen bonds, and thus with a lesser cost in terms of binding energy and $\mathrm{Km}$.

The analysis of MD simulations suggests that the CMY-136 structure may show increased flexibility around the R1 and R2 pockets in the active site. The R2 pocket is usually described as 
surrounded by the R2 loop (residues 289-307) and the proximal residues of helixes H10 and H11 1,19, and the R1 pocket is defined ad surrounded by the $\Omega$ loop (residues $178-226)^{1,19}$ (Fig. S6). The R1 substituent of ceftazidime, and other $\beta$-lactams, also interacts with other residues surrounding it, namely Q120, N152, residues 218-220 and S343 (Fig. S6). As previously proposed by other authors $^{9,20}$, an increased $k_{\text {cat }}$ may be a consequence of an increased protein flexibility, that may allow the acyl-enzyme complexes to more often adopt a conformation competent for being attacked by the hydrolytic water and be released. In the case of CMY-136, a change in the $\Omega$-loop conformation, widening the active site cavity by displacing V211, may also participate in the $k_{\text {cat }}$ increase observed for certain substrates. As observed by the superposition and RMSD analysis of the MD simulations, the CMY-136 $\Omega$-loop tends to adopt a conformation more similar to the one observed in the CMY-136 structure than to that observed in the CMY-2 structure, regardless of the starting conformation. For CMY-2 starting with the $\Omega$-loop in its native conformation, some fluctuation is observed but finally a conformation more similar to this native one than to the one in CMY-136 crystal structure is adopted. As mentioned earlier, this is not observed for the CMY-2 simulation starting with a CMY-136 $\Omega$-loop conformation. In this simulation the loop remains more flexible and the Y221 sidechain adopts different conformations, as observed in the case of CMY136. The stability of the native Y221 conformation (conserved in class C structures) in the CMY-2 MD simulation, and the fact that this conformation is not reached in a 10 ns simulation starting from a different conformation, suggests that the native conformation of the $\Omega$-loop of class $\mathrm{C}$ enzymes may be quite stable, and a relatively high energetic barrier might have to be overcome to reach, or come out of it. In contrast, H221 in CMY-136 easily adopts several conformations throughout the simulations, starting from either crystal structure conformations (CMY-2 and CMY-136). This in turn may help to explain the experimental data observed, i.e. a flexible H221 would allow CMY136 to hydrolyse $\beta$-lactams in spite of its conformation in the crystal structure. Were H221 to remain in this conformation, CMY-136 would most likely be incapable of interacting with its substrates, especially those with a more rigid, bulkier, ramified R1 sidechain. The fact that H221 
can adopt different conformations during the MD simulation, one of them similar to the Y221 conformation in CMY-2, helps to imagine how it may still accommodate the substrates in its active site cavity. On the other hand, this flexibility may also explain the increase in $\mathrm{Km}$ observed for most $\beta$-lactams. Although H221 seems to be much more flexible than Y221 and may adopt several conformations, once CMY-136 is interacting with the substrate, H221 would probably lose much of its movement freedom. This would imply an energetic cost, and an increase in $\mathrm{Km}$ for the reaction. Such increase in Km would clearly not be observed in the case of CMY-2 or other class C enzymes with Y221 in its native conformation, as it is probably static enough that no movement restrain is imposed on it upon binding of the substrate.

\section{CONCLUSION}

CMY-136 is a novel $\beta$-lactamase, which belongs to the CMY-2-like family. It possesses a single unusual mutation, $\mathrm{Y} 221 \mathrm{H}$, in a position highly conserved in all class $\mathrm{C} \beta$-lactamases. We compared here its genetic environment to that of the closest plasmid harbouring CMY-2 and characterized CMY-136 both biochemically and structurally. Finally, in silico modelling techniques suggested a possible structural explanation for the differences observed compared to CMY-2. These results allowed us to better assess the potential of class $C \beta$-lactamases for their spectrum extension and for their potential threat to public health. Finally, the identification of the $\mathrm{Y} 221 \mathrm{H}$ mutation in a natural variant of CMY-2 and which confers resistance to ceftolozane/tazobactam prior to the use of this latter antibiotic in clinics is worrisome, since it may indicate a more rapid appearance of resistance, as this type of enzyme may be selected.

\section{EXPERIMENTAL PROCEDURES}

\section{Bacterial strains}


bla $a_{\mathrm{CMY}-136}$ was recovered from a urinary tract E. coli isolate $\mathrm{EC} 13^{6} . b l a_{\mathrm{CMY}-2}$ was recovered from the E. cloacae ec-204 strain (Bicêtre strain collection). E. coli TOP10 (Invitrogen, Saint-Aubin, France) and E. coli BL21 (DE3) (Novagen, VWR International, Fontenay-sous-Bois, France) were used for cloning experiments and protein overproduction, respectively.

\section{Plasmid extraction and cloning}

Plasmid DNA from E. coli EC13 was extracted using the Kieser method ${ }^{7}$. The Kieser extracted DNA was used to transform E. coli TOP10 strain by electroporation. The electroporants were plated on TSA plate containing ampicillin $(100 \mu \mathrm{g} / \mathrm{ml})$. Transformants were analyzed by PCR using the primers CMY-2A and CMY-2B $\quad\left(5^{\prime}\right.$-aaaaacatatgatgaaaaaatcgttatgctgc-3' ${ }^{\prime}$ and 5'aaaaggatccttattgcagcttttcaagaatgc-3', respectively). From the transformants harbouring bla $a_{\mathrm{CMY}-2 \text {-like }}$ genes, plasmid DNA was extracted using Kieser's method and subsequently analysed on a $0.7 \%$ agarose gel stained with ethidium bromide. Plasmids of ca. 154, 66, 48, and $7 \mathrm{~kb}$ of E. coli NCTC 50192 were used as plasmid size markers ${ }^{21}$.

\section{Plasmid sequencing (WGS)}

Plasmid DNA was extracted from an E. coli TOP10 clone harbouring the wild type pCMY-136 plasmid using the QIAGEN Plasmid Maxi Kit following the manufacturer's instructions. The DNA concentration and purity were controlled by a Qubit ${ }^{\circledR} 2.0$ Fluorometer using the dsDNA HS and/or BR assay kit (Life technologies, Carlsbad, CA, US). The DNA library was prepared using the Nextera XT-v3 kit (Illumina, San Diego, CA, US) according to the manufacturer's instructions and then run on Miseq (Illumina) for generating paired-end 300-bp reads. De novo assembly was performed by CLC Genomics Workbench v9.5.3 (Qiagen, Hilden, Germany) after quality trimming (Qs $\geq 20)$ with word size 34 .

The acquired antimicrobial resistance genes were identified by uploading the assembled plasmid to the Resfinder server v2.1 (http://cge.cbs.dtu.dk/services/ResFinder-2.1 ${ }^{22}$. Plasmid incompatibility 
group was obtained by uploading the plasmid sequence to PlasmidFinder server 1.3 (https://cge.cbs.dtu.dk/services/PlasmidFinder/) $)^{23}$. Plasmid sequence was blasted (https://blast.ncbi.nlm.nih.gov/Blast.cgi) ${ }^{24}$ and compared to the best hit using Artemis Comparison Tool $^{25}$.

\section{Cloning of bla $a_{\mathrm{CMY}-136}$ and bla $_{\mathrm{CMY}-2}$ genes}

PCR amplification of bla $a_{\mathrm{CMY}-136}$ and $b l a_{\mathrm{CMY}-2}$ genes was performed using total DNA extraction of $E$. coli EC13 and Enterobacter cloacae ec-204, and primers CMY-2A (5'aaaaacatatgatgaaaaaatcgttatgctgc-3') and CMY-2B (5'-aaaaggatccttattgcagcttttcaagaatgc-3'). Amplicons were cloned into pCR ${ }^{\circledR}$-Blunt II-TOPO ${ }^{\circledR}$ cloning plasmid (Invitrogen, Illkirch, France) under regulation by the pLac promoter. The recombinant pTOPO-cmy-136 and pTOPO-cmy-2 plasmids were electroporated into the E. coli TOP10 strain and then selected using TSA-plate containing kanamycin $(50 \mathrm{mg} / \mathrm{L})$. bla $a_{\mathrm{CMY}-136}$ and $b l a_{\mathrm{CMY}-2}$ were amplified using primers INFcmy-2A (5'-aaggagatatacatatgatgaaaaatcgttatgct-3') and INFcmy-2B ggtggtggtgctcgaattgcagcttttcaagaatgc-3') and cloned into pET-41b(+) expression vector (Novagen, VWR International, Fontenay-sous-Bois, France) using the NEBuilder® HiFiDNA Assembly Cloning Kit (New England BioLabs ${ }^{\circledR} I n c$, United Kingdom), following the manufacturer's instructions. Recombinant plasmids pET41b-cmy-136 and pET41b-cmy-2 were electroporated into electrocompetent E. coli BL21 (DE3) and selected using TSA-plates containing kanamycin (50 $\mathrm{mg} / \mathrm{L})$.

Recombinant plasmids were extracted using the Qiagen miniprep kit and both strands of the inserts were sequenced using M13 F and M13 R primers, for the pCR ${ }^{\circledR}-$ Blunt II-TOPO ${ }^{\circledR}$ plasmid (Invitrogen, Illkirch, France), and T7 promoter and T7 terminator primers, for pET-41b(+) (Novagen, VWR International, Fontenay-sous-Bois, France), with an automated sequencer (ABI Prism 3100; Applied Biosystems, Les Ulis, France). The nucleotide sequences were analysed using 
software available at the National Center for Biotechnology Information website (http://www.ncbi.nlm.nih.gov).

\section{Antimicrobial agents, susceptibility testing and microbiological techniques}

Antimicrobial susceptibilities were determined by disk diffusion technique on Mueller-Hinton agar (Bio-Rad, Marnes-La-Coquette, France) and interpreted according to the EUCAST breakpoints, updated in 2015 (http://www.eucast.org). Minimal inhibitory concentration (MIC) values were determined using the Etest technique (BioMérieux, Paris, France).

\section{$\beta$-Lactamase purification}

Overnight cultures of E. coli BL21 (DE3) harbouring either pET41b-cmy-2 or pET41b-cmy-136 recombinant plasmids were used to inoculate $2 \mathrm{~L}$ of BHI broth containing $50 \mathrm{mg} / \mathrm{L}$ kanamycin. Bacteria were cultured at $37^{\circ} \mathrm{C}$ until reaching an $\mathrm{OD}$ of 0.6 at $600 \mathrm{~nm}$, and protein expression was induced overnight at $25^{\circ} \mathrm{C}$ with $0.2 \mathrm{mM}$ IPTG. Cultures were then centrifuged at $6000 \mathrm{~g}$ for $15 \mathrm{~min}$ and the pellets resuspended with $10 \mathrm{~mL}$ of Buffer A (20 mM PBS, $175 \mathrm{mM} \mathrm{K}_{2} \mathrm{SO}_{4}, 40 \mathrm{mM}$ imidazol, pH 7.40). Bacterial cells were disrupted by sonication and protein solution was clarified by centrifugation at $10,000 \mathrm{~g}$ for $1 \mathrm{~h}$ at $4^{\circ} \mathrm{C}$. The supernatant was then centrifuged at $48,000 \mathrm{~g}$ for 1 h at $4^{\circ} \mathrm{C}$. CMY-136 or CMY-2 was purified using a NTA-Nickel pseudo-affinity chromatography column (GE Healthcare, Freiburg, Germany). Elution was performed in a gradient of 0 to $100 \%$ Buffer B (20 mM PBS, $175 \mathrm{mM} \mathrm{K}_{2} \mathrm{SO}_{4}, 500 \mathrm{mM}$ imidazol, $\mathrm{pH}$ 7.40). Purity was assessed by SDSPAGE, and pure fractions were pooled and dialyzed against $100 \mathrm{mM}$ sodium phosphate buffer $(\mathrm{pH}$ 7.4) $50 \mathrm{mM}$ potassium sulphate and concentrated up to $6.4 \mathrm{mg} / \mathrm{ml}$ using Vivaspin ${ }^{\circledR}$ columns (GE Healthcare, Freiburg, Germany). Protein concentration was determined using Bradford Protein assay (Bio-Rad) ${ }^{26}$.

\section{Steady-state kinetic parameters}


Kinetic parameters of purified CMY-136 and CMY-2 were determined at 100mM sodium phosphate buffer ( $\mathrm{pH}$ 7.0), with extinction coefficients as detailed in Table S1. When available, published values from previous studies for CMY-2 were used for comparison with those determined for CMY-136 (Table 2). The $k_{\text {cat }}$ and $K \mathrm{~m}$ values were determined by analysing hydrolysis of $\beta$ lactams under initial-rate conditions with an ULTROSPEC 2000 model UV spectrophotometer (Amersham Pharmacia Biotech) using the Eadie-Hoffstee linearization of the Michaelis-Menten equation. The different $\beta$-lactams were purchased from Sigma-Aldrich (Saint-Quentin-Fallavier, France). Low Km values were determined as competitive inhibition constants, Ki, in the presence of a good reporter substrate (cephalothin) ${ }^{11}$.

\section{Protein crystallization and crystallography}

Initial crystallization screenings of CMY-136 were set up using the Mosquito ${ }^{\circledR}$ HTS (TTP LabTech) in four crystallization screening suites: Classics, AmSO4, PEGs and PEGs II (Qiagen/NeXtal). Plates were incubated at 293K in the ROCK IMAGER 1000 (Formulatrix, Inc). The crystal was transferred to a cryo-protectant solution consisting of the mother liquor supplemented with $25 \%$ glycerol and flash-frozen in liquid nitrogen.

Diffraction data was collected at $100 \mathrm{~K}$ in a nitrogen cryostream on the PROXIMA2 beamline at the SOLEIL synchrotron (Saint-Aubin, France). The data were indexed and integrated with $\mathrm{XDS}^{27}$ in the autoPROC toolbox ${ }^{28}$. Two incomplete datasets were merged to try to make as complete a dataset as possible, using autoPROC. Data scaling was performed using AIMLESS $^{29}$ from the CCP4 suite ${ }^{30}$. Data collection and refinement statistics are given in Table 4. The structure of CMY136 was solved by the molecular replacement method with Phaser ${ }^{31}$ using the structure of CMY-2 (PDB code 1ZC2) as a search model. The model was rebuilt manually in $\operatorname{Coot}^{32}$ and then refined using BUSTER-TNT ${ }^{33}$ with local noncrystallographic symmetry (NCS) restraints and a translationlibration-screw (TLS) description of $\mathrm{B}$ factors $^{34}$. The quality of the final refined model was assessed using MolProbity ${ }^{35}$. Crystal structure images were generated using PyMOL ${ }^{36}$. 


\section{Structure analysis, covalent complexes and molecular dynamics simulations}

CMY-136 structure comparison to the previously published CMY-2 structure (PDB code 1ZC2) and analysis of docking results were performed with UCSF Chimera package ${ }^{37}$.

Covalent complexes of CMY-2 and CMY-136 with ceftazidime, cefotaxime and cefuroxime were created by superposing the structures of CMY-2 and CMY-136 with the available structures of class C enzymes with ceftazidime (PDB code 1IEL) and cefotaxime (PDB code 4KG2), and modelling cefuroxime on the conformation of cefotaxime. Ligand structures were generated with 3D Structure Generator CORINA Classic (Molecular Networks GmbH, Nuremberg, Germany).

Molecular dynamics simulations of CMY-2 and CMY-136 were performed with Gromacs v4.6 ${ }^{37}$ using the OPLS-AA force field. ${ }^{38}$ The covalent ligands were parametrized using a modified version of our in house MOL2FF tool. Each system was energy-minimized until convergence using a steepest descents algorithm. Molecular dynamics with position restraints for $200 \mathrm{ps}$ was then performed followed by the production run of $10 \mathrm{~ns}$, which was repeated three times to ensure the reproducibility. During the position restraints and production runs, the Parinello-Rahman method was used for pressure coupling, and the temperature was coupled using the Nosé-Hoover method at 300 K. Electrostatics were calculated with the particle mesh Ewald method. The P-LINCS algorithm was used to constrain bond lengths, and a time step of $2 \mathrm{fs}$ was used throughout.

\section{Nucleotide sequences accession numbers and PDB deposition.}

The amino acid sequence of $b l a_{\mathrm{CMY}-136}$ gene and the nucleotide sequence of its natural plasmid pCMY-136 have been submitted to the EMBL/Genbank nucleotide sequence database under the accession numbers AVR61040.1 and MG844436.1, respectively. The crystallographic structure of CMY-136 has been deposited to the PDB, accession code 6G9T. Authors will release the atomic coordinates and experimental data upon article publication. 


\section{AKNOWLEDGEMENTS}

We acknowledge SOLEIL for provision of synchrotron radiation facilities (proposal ID BAG20150780) in using PROXIMA beamlines. This work was supported by the Laboratory of Excellence in Research on Medication and Innovative Therapeutics (LERMIT) [grant number ANR-10-LABX-33], by the JPIAMR transnational project DesInMBL [grant number ANR-14JAMR-0002] and by the Région Ile-de-France (DIM Malinf).

\section{CONFLICT OF INTEREST}

The authors declare that they have no conflicts of interests with the content of this article.

Supporting Information. Sequence alignment of CMY-2 and CMY-136 (Fig. S1), chemical structures of $\beta$-lactams and $\beta$-lactamase inhibitors used in this study (Fig. S2), different conformations of Y150 in the CMY-2 and CMY-136 structures (Fig. S3), RMSD of the MD simulations (Fig. S4), RMSD analysis of $\Omega$-loop from MD trajectories (Fig. S5), R1 and R2 pockets in CMY-2 (Fig. S6) and extinction coefficients and wavelengths for $\beta$-lactams (Table S1).

\section{REFERENCES}

(1) Jacoby, G. A. (2009) AmpC Beta-Lactamases. Clin. Microbiol. Rev., 22 (1), 161-182 DOI 10.1128/CMR.00036-08.

(2) Fang, L.-X.; Sun, J.; Li, L.; Deng, H.; Huang, T.; Yang, Q.-E.; Li, X.; Chen, M.-Y.; Liao, X.-P.; Liu, Y.-H. (2015) Dissemination of the Chromosomally Encoded CMY-2 Cephalosporinase Gene in Escherichia Coli Isolated from Animals. Int. J. Antimicrob. Agents, 46 (2), 209-213 DOI 10.1016/j.ijantimicag.2015.04.003.

(3) Naas, T.; Oueslati, S.; Bonnin, R. A.; Dabos, M. L.; Zavala, A.; Dortet, L.; Retailleau, P.; Iorga, B. I. (2017) Beta-Lactamase Database (BLDB) - Structure and Function. J. Enzyme Inhib. Med. Chem., 32 (1), 917-919 DOI 10.1080/14756366.2017.1344235. 
(4) Powers, R. A. (2016) Structural and Functional Aspects of Extended-Spectrum AmpC Cephalosporinases. Curr. Drug Targets, 17 (9), 1051-1060 DOI $10.2174 / 1573399811666150615144707$.

(5) Dahyot, S.; Broutin, I.; de Champs, C.; Guillon, H.; Mammeri, H. (2013) Contribution of Asparagine 346 Residue to the Carbapenemase Activity of CMY-2 $\beta$-Lactamase. FEMS Microbiol. Lett., 345 (2), 147-153 DOI 10.1111/1574-6968.12199.

(6) Mammeri, H.; Poirel, L.; Fortineau, N.; Nordmann, P. (2006) Naturally Occurring ExtendedSpectrum Cephalosporinases in Escherichia Coli. Antimicrob. Agents Chemother., 50 (7), 2573-2576 DOI 10.1128/AAC.01633-05.

(7) Kieser, T. (1984) Factors Affecting the Isolation of CCC DNA from Streptomyces Lividans and Escherichia Coli. Plasmid, 12 (1), 19-36 DOI 10.1016/0147-619X(84)90063-5.

(8) Cao, G.; Allard, M. W.; Hoffmann, M.; Monday, S. R.; Muruvanda, T.; Luo, Y.; Payne, J.; Rump, L.; Meng, K.; Zhao, S.; et al. (2015) Complete Sequences of Six IncA/C Plasmids of Multidrug-Resistant Salmonella Enterica Subsp. Enterica Serotype Newport. Genome Announc., 3 (1), e00027-15 DOI 10.1128/genomeA.00027-15.

(9) Kotsakis, S. D.; Miriagou, V.; Vetouli, E. E.; Bozavoutoglou, E.; Lebessi, E.; Tzelepi, E.; Tzouvelekis, L. S. (2015) Increased Hydrolysis of Oximino- $\beta$-Lactams by CMY-107, a Tyr199Cys Mutant Form of CMY-2 Produced by Escherichia Coli. Antimicrob. Agents Chemother., 59 (12), 7894-7898 DOI 10.1128/AAC.01793-15.

(10) Endimiani, A.; Doi, Y.; Bethel, C. R.; Taracila, M.; Adams-Haduch, J. M.; O’Keefe, A.; Hujer, A. M.; Paterson, D. L.; Skalweit, M. J.; Page, M. G. P.; et al. (2010) Enhancing Resistance to Cephalosporins in Class C Beta-Lactamases: Impact of Gly214Glu in CMY-2. Biochemistry, 49 (5), 1014-1023 DOI 10.1021/bi9015549.

(11) Bauvois, C.; Ibuka, A. S.; Celso, A.; Alba, J.; Ishii, Y.; Frère, J.-M.; Galleni, M. (2005) Kinetic Properties of Four Plasmid-Mediated AmpC Beta-Lactamases. Antimicrob. Agents Chemother., 49 (10), 4240-4246 DOI 10.1128/AAC.49.10.4240-4246.2005. 
(12) Dahyot, S.; Mammeri, H. (2012) Hydrolysis Spectrum Extension of CMY-2-like $\beta$ Lactamases Resulting from Structural Alteration in the Y-X-N Loop. Antimicrob. Agents Chemother., 56 (3), 1151-1156 DOI 10.1128/AAC.05630-11.

(13) Skalweit, M. J.; Li, M.; Conklin, B. C.; Taracila, M. A.; Hutton, R. A. (2013) N152G, -S, and -T Substitutions in CMY-2 $\beta$-Lactamase Increase Catalytic Efficiency for Cefoxitin and Inactivation Rates for Tazobactam. Antimicrob. Agents Chemother., 57 (4), 1596-1602 DOI 10.1128/AAC.01334-12.

(14) Kotsakis, S. D.; Papagiannitsis, C. C.; Tzelepi, E.; Tzouvelekis, L. S.; Miriagou, V. (2009) Extended-Spectrum Properties of CMY-30, a Val211Gly Mutant of CMY-2 Cephalosporinase. Antimicrob. Agents Chemother., 53 (8), 3520-3523 DOI 10.1128/AAC.00219-09.

(15) Kusumoto, M.; Ooka, T.; Nishiya, Y.; Ogura, Y.; Saito, T.; Sekine, Y.; Iwata, T.; Akiba, M.; Hayashi, T. (2011) Insertion Sequence-Excision Enhancer Removes Transposable Elements from Bacterial Genomes and Induces Various Genomic Deletions. Nat. Commun., 2 (1), 152 DOI 10.1038/ncomms1152.

(16) Lefurgy, S. T.; Malashkevich, V. N.; Aguilan, J. T.; Nieves, E.; Mundorff, E. C.; Biju, B.; Noel, M. A.; Toro, R.; Baiwir, D.; Papp-Wallace, K. M.; et al. (2016) Analysis of the Structure and Function of FOX-4 Cephamycinase. Antimicrob. Agents Chemother., 60 (2), 717-728 DOI 10.1128/AAC.01887-15.

(17) Thomas, V. L.; McReynolds, A. C.; Shoichet, B. K. (2010) Structural Bases for StabilityFunction Tradeoffs in Antibiotic Resistance. J. Mol. Biol., 396 (1), 47-59 DOI 10.1016/j.jmb.2009.11.005.

(18) Berrazeg, M.; Jeannot, K.; Ntsogo Enguéné, V. Y.; Broutin, I.; Loeffert, S.; Fournier, D.; Plésiat, P. (2015) Mutations in $\beta$-Lactamase AmpC Increase Resistance of Pseudomonas Aeruginosa Isolates to Antipseudomonal Cephalosporins. Antimicrob. Agents Chemother., 59 (10), 6248-6255 DOI 10.1128/AAC.00825-15. 
(19) A. Powers, R. (2016) Structural and Functional Aspects of Extended-Spectrum AmpC Cephalosporinases. Curr. Drug Targets, 17 (9), 1051-1060 DOI $10.2174 / 1573399811666150615144707$.

(20) Kotsakis, S. D.; Tzouvelekis, L. S.; Petinaki, E.; Tzelepi, E.; Miriagou, V. (2011) Effects of the Val211Gly Substitution on Molecular Dynamics of the CMY-2 Cephalosporinase: Implications on Hydrolysis of Expanded-Spectrum Cephalosporins. Proteins Struct. Funct. Bioinforma., 79 (11), 3180-3192 DOI 10.1002/prot.23150.

(21) Dortet, L.; Oueslati, S.; Jeannot, K.; Tandé, D.; Naas, T.; Nordmann, P. (2015) Genetic and Biochemical Characterization of OXA-405, an OXA-48-Type Extended-Spectrum $\beta$ Lactamase without Significant Carbapenemase Activity. Antimicrob. Agents Chemother., 59 (7), 3823-3828 DOI 10.1128/AAC.05058-14.

(22) Zankari, E.; Hasman, H.; Cosentino, S.; Vestergaard, M.; Rasmussen, S.; Lund, O.; Aarestrup, F. M.; Larsen, M. V. (2012) Identification of Acquired Antimicrobial Resistance Genes. J. Antimicrob. Chemother., 67 (11), 2640-2644 DOI 10.1093/jac/dks261.

(23) Carattoli, A.; Zankari, E.; García-Fernández, A.; Voldby Larsen, M.; Lund, O.; Villa, L.; Møller Aarestrup, F.; Hasman, H. (2014) In Silico Detection and Typing of Plasmids Using PlasmidFinder and Plasmid Multilocus Sequence Typing. Antimicrob. Agents Chemother., 58 (7), 3895-3903 DOI 10.1128/AAC.02412-14.

(24) Altschul, S. F.; Gish, W.; Miller, W.; Myers, E. W.; Lipman, D. J. (1990) Basic Local Alignment Search Tool. J. Mol. Biol., 215 (3), 403-410 DOI 10.1016/S00222836(05)80360-2.

(25) Carver, T. J.; Rutherford, K. M.; Berriman, M.; Rajandream, M.-A.; Barrell, B. G.; Parkhill, J. (2005) ACT: The Artemis Comparison Tool. Bioinformatics, 21 (16), 3422-3423 DOI 10.1093/bioinformatics/bti553.

(26) Bradford, M. M. (1976) A Rapid and Sensitive Method for the Quantitation of Microgram Quantities of Protein Utilizing the Principle of Protein-Dye Binding. Anal. Biochem., 72, 
$248-254$.

(27) Kabsch, W. (2010) Integration, Scaling, Space-Group Assignment and Post-Refinement. Acta Crystallogr. D. Biol. Crystallogr., 66 (Pt 2), 133-144 DOI

$10.1107 / \mathrm{S} 0907444909047374$.

(28) Vonrhein, C.; Flensburg, C.; Keller, P.; Sharff, A.; Smart, O.; Paciorek, W.; Womack, T.;

Bricogne, G. (2011) Data Processing and Analysis with the AutoPROC Toolbox. Acta

Crystallogr. Sect. D Biol. Crystallogr., 67 (4), 293-302 DOI 10.1107/S0907444911007773.

(29) Evans, P. R.; Murshudov, G. N. (2013) How Good Are My Data and What Is the

Resolution? Acta Crystallogr. D. Biol. Crystallogr., 69 (Pt 7), 1204-1214 DOI

10.1107/S0907444913000061.

(30) Winn, M. D.; Ballard, C. C.; Cowtan, K. D.; Dodson, E. J.; Emsley, P.; Evans, P. R.;

Keegan, R. M.; Krissinel, E. B.; Leslie, A. G. W.; McCoy, A.; et al. (2011) Overview of the CCP4 Suite and Current Developments. Acta Crystallogr. D. Biol. Crystallogr., 67 (Pt 4), 235-242 DOI 10.1107/S0907444910045749.

(31) McCoy, A. J.; Grosse-Kunstleve, R. W.; Adams, P. D.; Winn, M. D.; Storoni, L. C.; Read, R. J. (2007) Phaser Crystallographic Software. J. Appl. Crystallogr., 40 (4), 658-674 DOI $10.1107 / \mathrm{S} 0021889807021206$.

(32) Emsley, P.; Lohkamp, B.; Scott, W. G.; Cowtan, K. (2010) Features and Development of Coot. Acta Crystallogr. Sect. D Biol. Crystallogr., 66 (4), 486-501 DOI 10.1107/S0907444910007493.

(33) Bricogne, G.; Blanc, E.; Brandl, M.; Flensburg, C.; Keller, P.; Paciorek, W.; Roversi, P.; Sharff, A.; Smart, O. S.; Vonrhein, C.; et al. (2011) AutoBUSTER, Version 1.6. 0. Glob. Phasing Ltd, Cambridge, UK.,.

(34) Murshudov, G. N.; Skubák, P.; Lebedev, A. A.; Pannu, N. S.; Steiner, R. A.; Nicholls, R. A.; Winn, M. D.; Long, F.; Vagin, A. A. (2011) REFMAC 5 for the Refinement of Macromolecular Crystal Structures. Acta Crystallogr. Sect. D Biol. Crystallogr., 67 (4), 
355-367 DOI 10.1107/S0907444911001314.

(35) Chen, V. B.; Arendall, W. B.; Headd, J. J.; Keedy, D. A.; Immormino, R. M.; Kapral, G. J.; Murray, L. W.; Richardson, J. S.; Richardson, D. C. (2010) MolProbity: All-Atom Structure Validation for Macromolecular Crystallography. Acta Crystallogr. Sect. D Biol.

Crystallogr., 66 (1), 12-21 DOI 10.1107/S0907444909042073.

(36) Schrödinger, L. The PyMOL Molecular Graphics System, Version 1.8.

(37) Pettersen, E. F.; Goddard, T. D.; Huang, C. C.; Couch, G. S.; Greenblatt, D. M.; Meng, E. C.; Ferrin, T. E. (2004) UCSF Chimera - A Visualization System for Exploratory Research and Analysis. J. Comput. Chem., 25 (13), 1605-1612 DOI 10.1002/jcc.20084. 


\section{For Table of Contents Use Only}

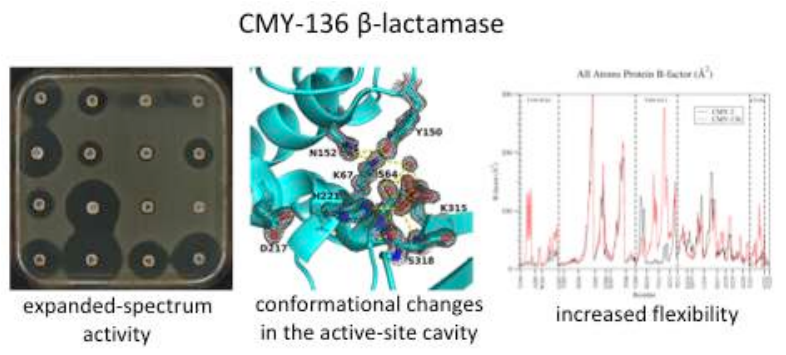




\title{
Supplementary Information
}

\section{Genetic, biochemical and structural characterization of CMY-136 $\beta$-lactamase, a peculiar CMY-2 variant}

\author{
Agustin Zavala $^{1,2}$, Pascal Retailleau ${ }^{1}$, Eddy Elisée ${ }^{1}$, Bogdan I. Iorga $^{1^{*}}$, Thierry Naas ${ }^{2,3,4,5^{*}}$ \\ ${ }^{1}$ Institut de Chimie des Substances Naturelles, CNRS UPR 2301, Université Paris-Saclay, LabEx \\ LERMIT, 1 avenue de la Terrasse, Bât. 27, 91198 Gif-sur-Yvette, France \\ ${ }^{2}$ EA7361 "Structure, dynamic, function and expression of broad spectrum $\beta$-lactamases", \\ Université Paris Sud, Université Paris Saclay, LabEx LERMIT, Faculty of Medicine, 78 rue du \\ Général Leclerc, 94275 Le Kremlin-Bicêtre, France \\ ${ }^{3}$ Bacteriology-Hygiene unit, Assistance Publique/Hôpitaux de Paris, Bicêtre Hospital, 78 rue du \\ Général Leclerc, 94275 Le Kremlin-Bicêtre, France \\ ${ }^{4}$ Associated French National Reference Center for Antibiotic Resistance: Carbapenemase- \\ producing Enterobacteriaceae, 78 rue du Général Leclerc, 94275 Le Kremlin-Bicêtre, France \\ ${ }^{5}$ Evolution and Ecology of Resistance to Antibiotics Unit, Institut Pasteur - APHP -Université \\ Paris Sud, 25-28 Rue du Dr Roux, 75015 Paris, France \\ *To whom correspondence should be addressed: \\ Bogdan I. Iorga: Institut de Chimie des Substances Naturelles, CNRS UPR 2301, 91198 Gif-sur- \\ Yvette, France. E-mail: bogdan.iorga@cnrs.fr; Tel. +33 1698230 94; Fax. +33 169077247 \\ Thierry Naas : Service de Bactériologie-Hygiène, Hôpital de Bicêtre, 78 rue du Général Leclerc, \\ 94275 Le Kremlin-Bicêtre, France. E-mail: thierry.naas@aphp.fr; Tel: +33 1452120 19; Fax: +33 \\ 145216340
}

Content Page

Fig. S1: Sequence alignment of CMY-2 and CMY-136 S2

Fig. S2: Chemical structures of $\beta$-lactams and $\beta$-lactamase inhibitors used in this study $\quad$ S3

Fig. S3: Different conformations of Y150 in the CMY-2 and CMY-136 structures $\quad$ S4

Fig. S4: RMSD of the MD simulations $\quad$ S5

Fig. S5: RMSD analysis of $\Omega$-loop from MD trajectories $\quad$ S6

Fig. S6: R1 and R2 pockets in CMY-2

Table S1: Extinction coefficients and wavelengths for $\beta$-lactams $\quad$ S8 
$C M Y-2$

CMY-2

CMY-136 $\alpha 1$ elelelelelelelele

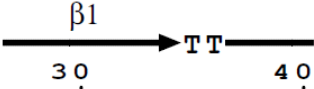

30

0 MMKK S L C CA L L T TASFS TFAAAKTEQQ IAD IVNRT I T P LMQEQA I P GMAVAVIYQGKPYY MMKK S LCCALLLTASF STFAAAKTEQQIAD I VNRT I T P LMOEQA I P GMAVAVI YQGKP YY

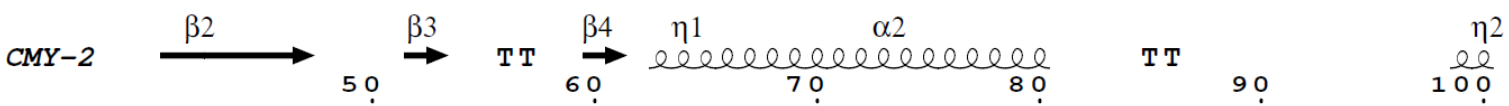

CMY-2 FTWGKADIANNHPVTQQTLEELGSVSKTFNGVLGGDA IARGEIKLSDPVTKYWPELTGKO

CMY-136 FTKGKADIANNHPVTQOTLEELGSVSKTFNGVLGGDAIARGEIKLSDPVTKYWPELTGKO

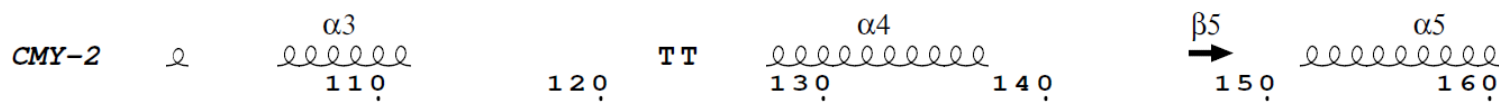

CMY-2

CMY-136 WOGIRLLHLATYTAGGLPLQIPDDVRDKAALLHFYQNWQPQWTPGAKRLYANSSIG LFGA

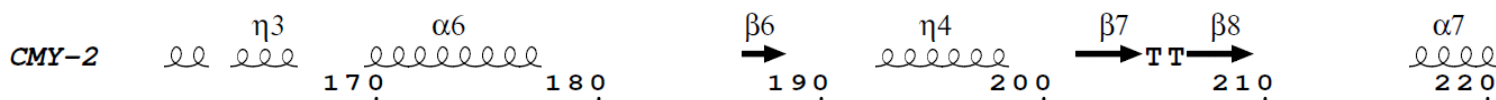

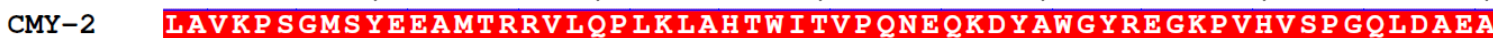

CMY-136 LAVKP SGMSYEEAMTRRVLQPLKLAHTWITVPQNEQKDYAKGYREGKPVHVSPGQLDAEA

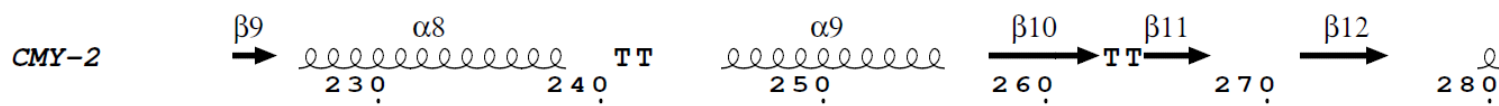

CMY-2 YGVKSSVIDMARWVQANMDASHVQEKTLQQGIALAQSRYWRI GDMYQGLGWEMLNWP LKA

CMY-136 HGVKS SVIDMARWVQANMDASHVQEKTLQQG I LAQSRYKR I GDMYQG L GK EMLNWP LKA

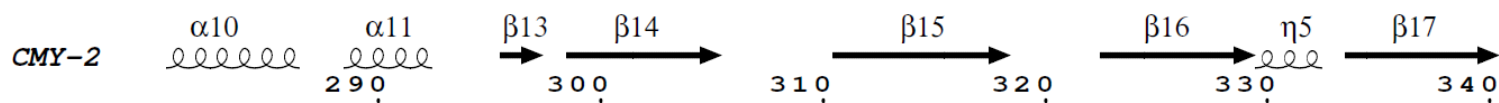

CMY-2 DSI INGSDSKVALAALPAVEVNP PAPAVKA SWVHKTGST GGF GSYVAFVP EKNLGIVMLA

CMY-136 DSIINGSDSKVALAALPAVEVNP PAPAVKASWVHKTGSTGGFGSYVAFVPEKNLGIVMLA

\begin{tabular}{lc} 
& \multicolumn{2}{c}{$\alpha 12$} \\
CMY-2 & eleelelelele \\
& 350 \\
CMY-2 & NKSYPNPVRVEAAKRILEKLQ \\
CMY-136 & NKSYPNPVRVEAAKRILEKLQ \\
\cline { 2 - 2 }
\end{tabular}

Fig. S1 Sequence alignment of CMY-2 and CMY-136. Sequence alignment of CMY-2 and CMY-136 showing the position of the mutated residue and secondary structure elements as defined for CMY-2 (PDB code 1ZC2). 


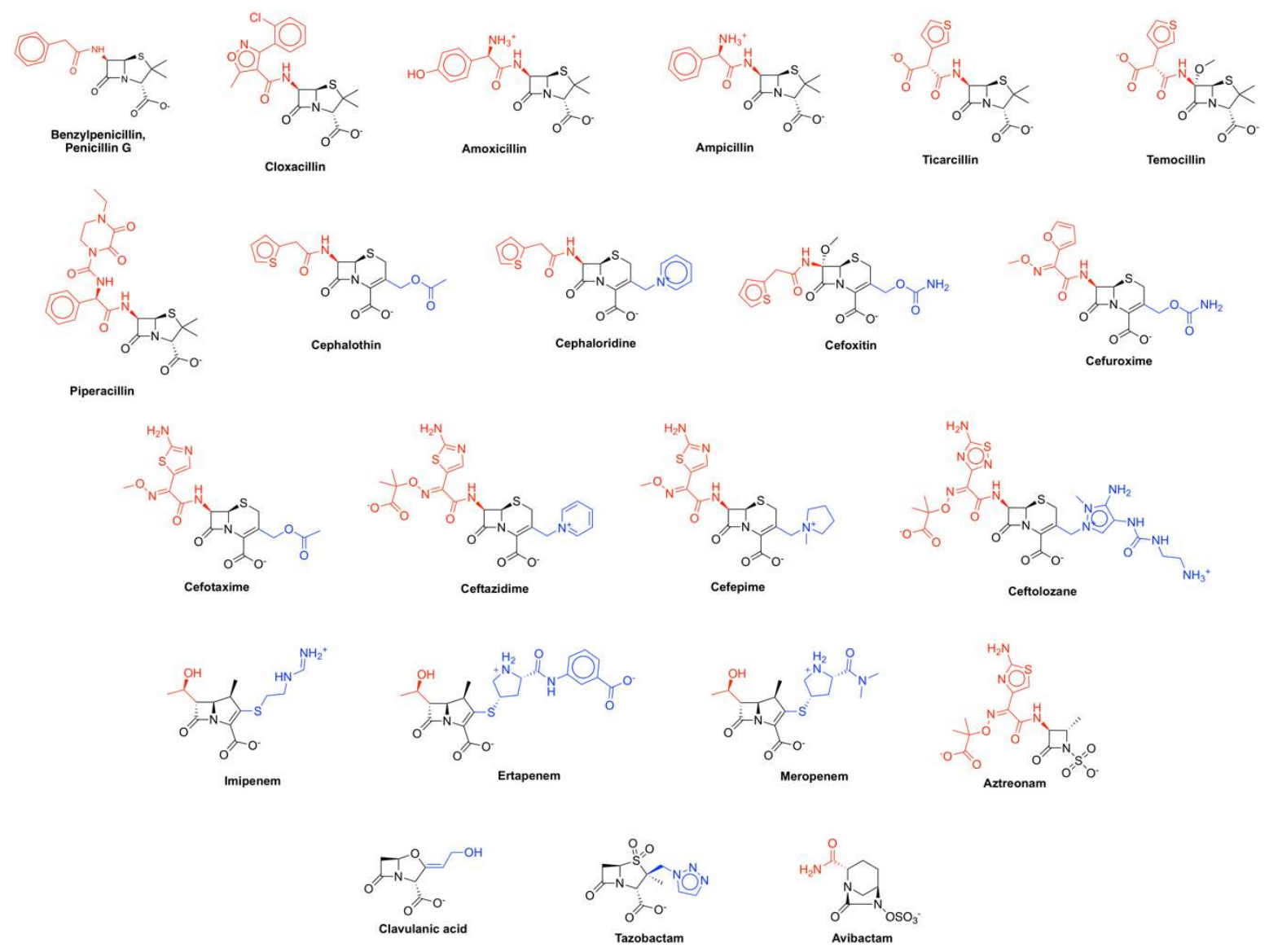

Fig. S2 Chemical structures of $\beta$-lactams and $\beta$-lactamase inhibitors used in this study. The chemical structures of the $\beta$-lactam antibiotics or $\beta$-lactamase inhibitors mentioned in this study (MICs, enzyme kinetics or molecular modelling experiments) are shown here. The R1 and R2 substituents are colored in red and blue, respectively. 


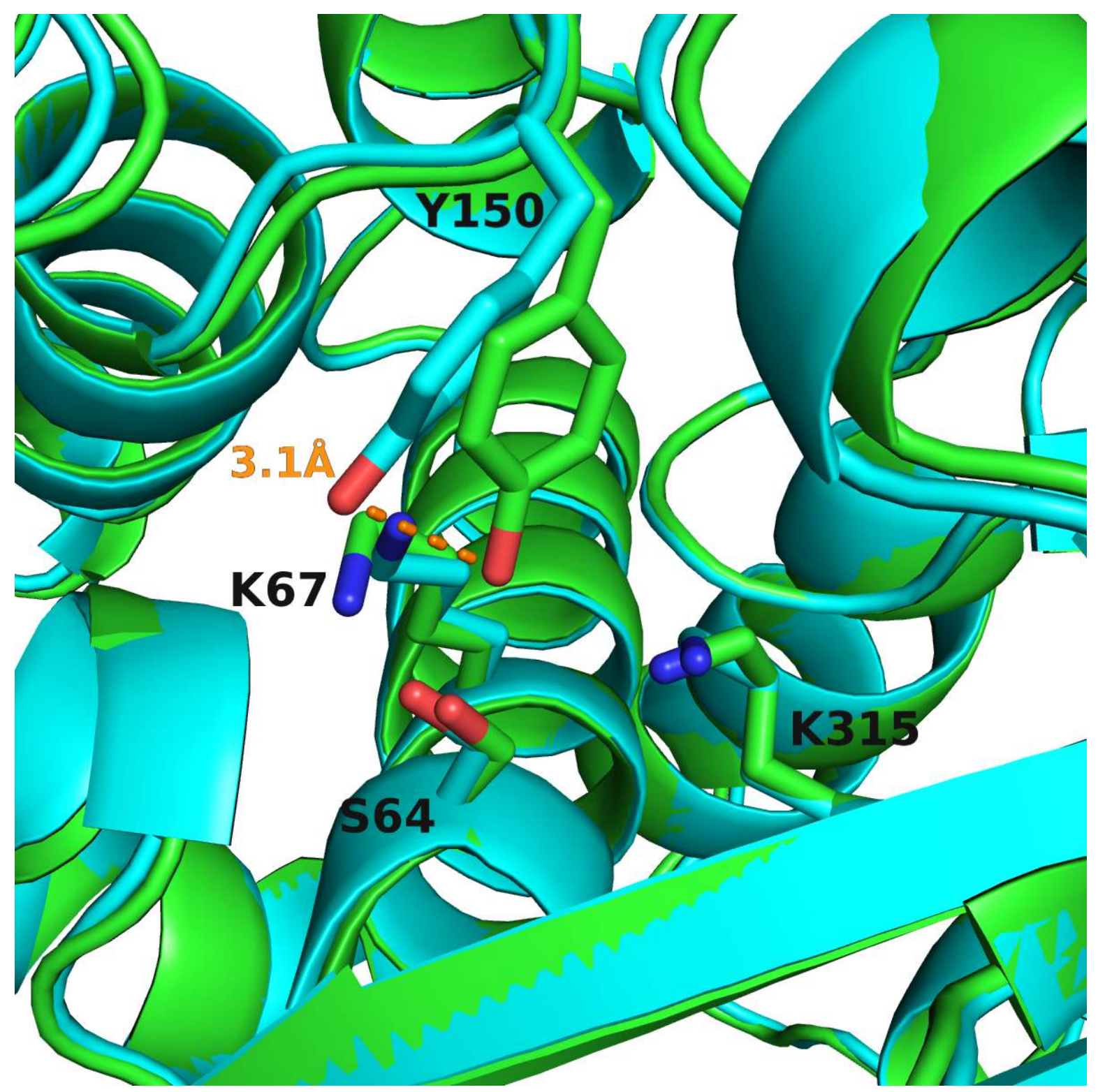

Fig. S3 Different conformations of Y150 in the CMY-2 and CMY-136 structures. Superposition of CMY-2 (green) and CMY-136 (cyan) structures shows that the conformation adopted by Y150, a mechanistically important residue, in the CMY-136 structure differs from that observed in CMY-2, with a shift of $3.1 \AA$ in its hydroxyl position. Other important residues are shown for reference. 
a

RMS Deviation

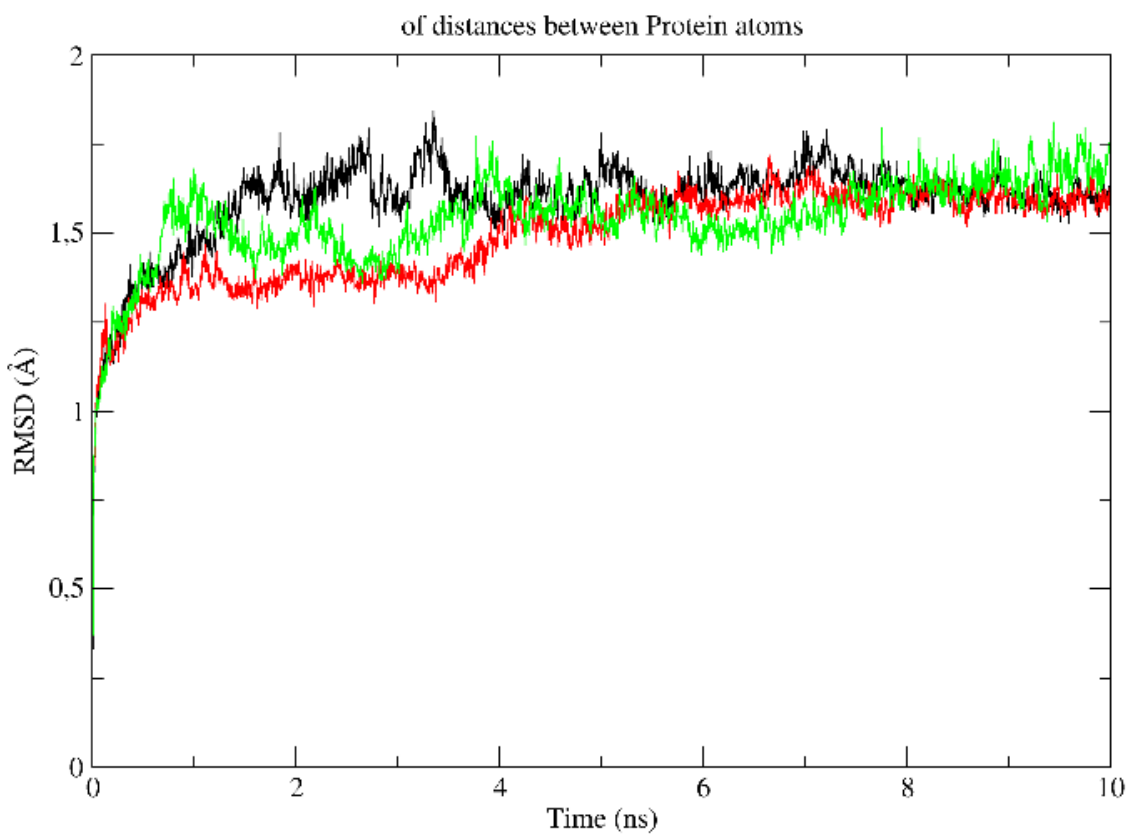

b

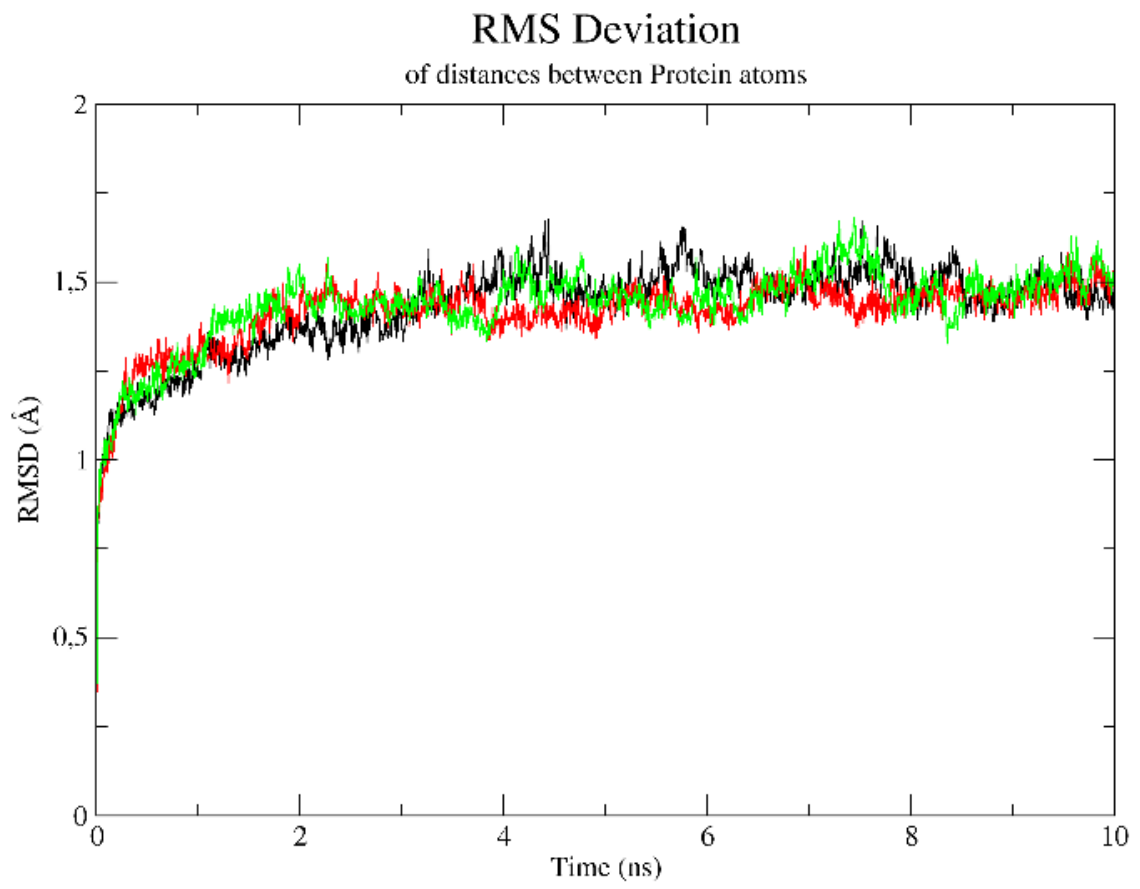

Fig. S4 RMSD of the MD simulations. RMSD of the 10 ns simulations of CMY-2 (A) and CMY136 (B) carried out in triplicate. 


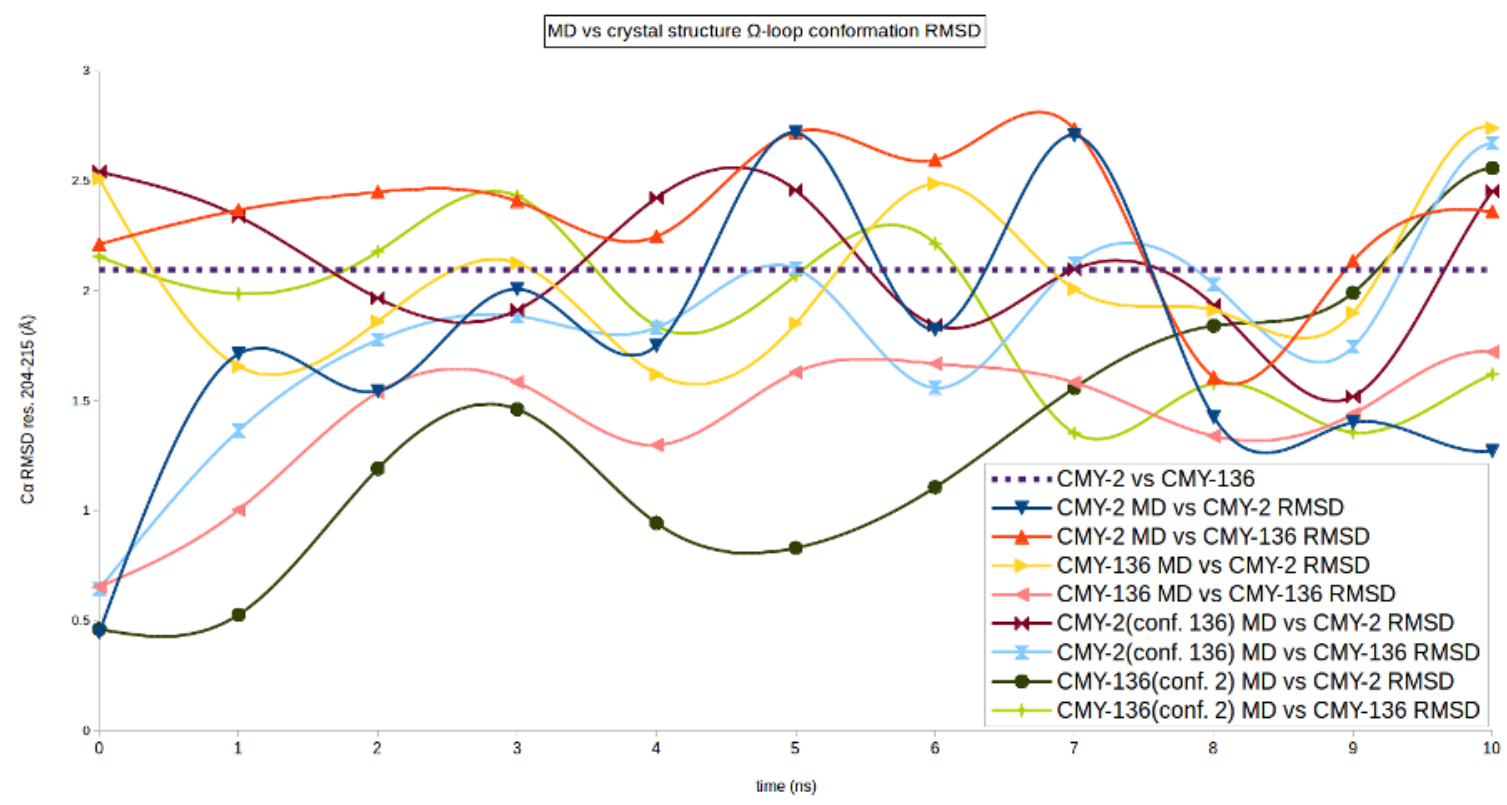

Fig. S5. RMSD analysis of $\Omega$-loop from MD trajectories. $\Omega$-loop $\mathrm{C} \alpha \mathrm{RMSD}$ analysis of the MD trajectories compared to CMY-2 and CMY-136 crystal structures. RMSD was calculated between crystal structure $\Omega$-loops, and MD simulation snapshots taken every 1 ns. Regardless of starting conformation, CMY-136 structures tend to converge to a conformation closer to that of CMY-136 crystal structure, and far from that of CMY-2 crystal structure. For CMY-2 simulations, when starting from the native structure conformation, $\Omega$-loop conformation is maintained closer to it than to that of CMY-136 $\Omega$-loop crystal structure. When starting from a CMY-136 $\Omega$-loop conformation, however, CMY-2 $\Omega$-loop conformation does not converge towards its own $\Omega$-loop crystal structure conformation. $\Omega$-loop RMSD between both crystal structures is represented by the purple dashed line. "CMY-2 MD": simulations for the CMY-2 structure starting from its own crystal structure conformation; "CMY-136 MD": simulations for the CMY-136 structure starting from its own crystal structure conformation; "CMY-2(conf. 136) MD": simulations for the CMY-2 structure starting with the $\Omega$-loop modelled on the CMY-136 crystal structure $\Omega$-loop conformation; "CMY136(conf. 2) MD”: simulations for the CMY-136 structure starting with the $\Omega$-loop modelled on the CMY-2 crystal structure $\Omega$-loop conformation. 


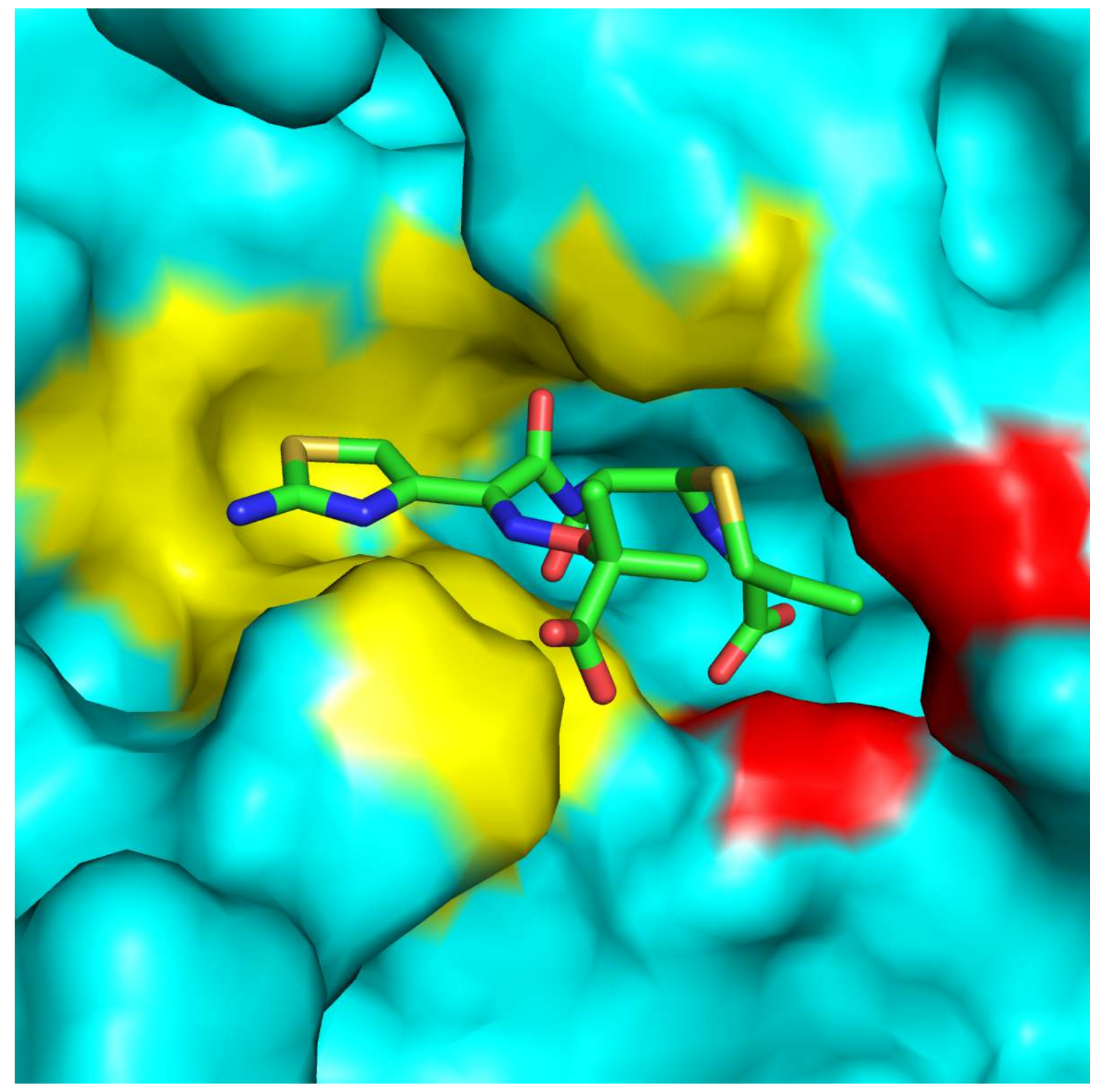

Fig. S6 R1 and R2 pockets in CMY-2. Surface representation of CMY-2 showing the different parts of the protein defining the R1 and R2 pockets. The R2 pocket (colored in red) is usually defined as surrounded by the R2 loop (residues 289-307) and the proximal parts of helixes H10 and H11. The R1 pocket (colored in yellow) is usually defined as surrounded by the $\Omega$ loop (residues 178-226). Apart from this, the R1 substituent of ceftazidime can interact with other surrounding residues: Q120, N152, S343 and residues 318-320. For reference, ceftazidime is shown as green sticks. 
Table S1 Extinction coefficients and wavelengths for $\boldsymbol{\beta}$-lactams. Extinction coefficients and wavelengths used for the different substrates in the kinetic parameter determinations.

\begin{tabular}{lcc}
\hline \multicolumn{1}{c}{ Substrate } & Wavelength $(\mathrm{nm})$ & $\Delta \varepsilon\left(\mathrm{M}^{-1} \mathrm{~cm}^{-1}\right)$ \\
\hline Benzylpenicillin & 232 & 1,100 \\
Cloxacillin & 220 & 700 \\
Ampicillin & 235 & 1,050 \\
Ticarcillin & 235 & 1,050 \\
Cefaloridine & 255 & 9,360 \\
Cefalotin & 262 & 7,960 \\
Cefoxitin & 265 & 7,380 \\
Cefuroxime & 262 & 7,800 \\
Cefotaxime & 265 & 6,260 \\
Ceftazidime & 260 & 8,660 \\
Cefepime & 264 & 8,240 \\
Ceftolozane & 259 & 1,900 \\
Imipenem & 297 & 9,210 \\
Aztreonam & 318 & 640 \\
\hline
\end{tabular}

